\title{
Imprints of magnetic power and helicity spectra on radio polarimetry statistics
}

\begin{abstract}
H. Junklewitz ${ }^{1}$ and T. A. Enßlin ${ }^{1}$
Max-Planck-Institut für Astrophysik, Karl-Schwarzschildstr. 1, 85741 Garching, Germany

e-mail: henrikju@mpa-garching.mpg.de

Received 6 August 2010 / Accepted 1 February 2011

ABSTRACT

The statistical properties of turbulent magnetic fields in radio-synchrotron sources should be imprinted on the statistics of polarimetric observables. In search of these imprints, i.e. characteristic modifications of the polarimetry statistics caused by magnetic field properties, we calculate correlation and cross-correlation functions from a set of observables that contain total intensity $I$, polarized intensity $P$, and Faraday depth $\phi$. The correlation functions are evaluated for all combinations of observables up to fourth order in magnetic field $\boldsymbol{B}$. We derive these analytically as far as possible and from first principles using only some basic assumptions, such as Gaussian statistics for the underlying magnetic field in the observed region and statistical homogeneity. We further assume some simplifications to reduce the complexity of the calculations, because for a start we were interested in a proof of concept. Using this statistical approach, we show that it is possible to gain information about the helical part of the magnetic power spectrum via the correlation functions $\left\langle P\left(\boldsymbol{k}_{\perp}\right) \phi\left(\boldsymbol{k}_{\perp}^{\prime}\right) \phi\left(\boldsymbol{k}_{\perp}^{\prime \prime}\right)\right\rangle_{\boldsymbol{B}}$ and $\left\langle I\left(\boldsymbol{k}_{\perp}\right) \phi\left(\boldsymbol{k}_{\perp}^{\prime}\right) \phi\left(\boldsymbol{k}_{\perp}^{\prime \prime}\right)\right\rangle_{\boldsymbol{B}}$. Using this insight, we construct an easy-to-use test for helicity called LITMUS (Local Inference Test for Magnetic fields which Uncovers heliceS), which gives a spectrally integrated measure of helicity. For now, all calculations are given in a Faraday-free case, but set up so that Faraday rotational effects can be included later.
\end{abstract}

Key words. magnetic fields - methods: data analysis - methods: statistical

\section{Introduction}

Magnetic fields are observed in almost all astronomical objects, among which we find planets and stars as well as galaxies and clusters. Most, if not all, of the interstellar and intergalactic plasma appear to be magnetized, and the magnetic fields contribute significantly to physical processes. Examples include the formation of stars (Price et al. 2009), the anisotropy of transport processes (thermal conduction or plasma resistivity, see e.g. Narayan \& Medvedev 2001), the angular momentum transport in accretion disks or the propagation of cosmic ray populations (Strong et al. 2007).

Although magnetic fields are ubiquitous in the cosmos, we often cannot treat them properly in astrophysical situations owing to our lack of knowledge of their properties. Cosmic magnetic fields are difficult to observe, and their distribution, evolution, and origins are far from perfectly understood. We have three main sources of information: the Zeeman effect, synchrotron radiation, and Faraday rotation. The Zeeman effect is extremely difficult to detect, because other line - shifting effects, such as thermal Dopplerbroadening, are usually stronger. We obtain a great deal of information from synchrotron radiation, but only regarding the magnetic field component perpendicular to the line of sight. To get a picture of the 3D magnetic field, one needs another source of information. This leads us to Faraday rotation, the change in the polarization plane of long wavelength radiation due to a magnetic field along the line of sight. Faraday rotation provides a powerful tool, but is also difficult to observe, to evaluate, and to interpret because ofe the involved line-of-sight projection. This projection is one of the main obstacles to understanding the 3D properties of magnetic fields.

One important property of cosmic magnetic fields that we do not know much about is magnetic helicity. It is defined as the integral

$H=\int_{V} \boldsymbol{A} \cdot \boldsymbol{B} \mathrm{d} x^{3}$

over a volume $V$ with surface $\partial V$ on which $\boldsymbol{n} \cdot \boldsymbol{B}=0$; where $A$ refers to the vector potential from electrodynamics with $\boldsymbol{B}=\nabla \times \boldsymbol{A}$. Helicity is a measure for the "spiral quality" of a magnetic field. It quantifies how much the magnetic field lines are sheared and twisted and counts the number of spirals the field lines exhibit within a given volume. Particularly turbulent magnetic fields should show considerable helicity. The relevance of helicity has increased since its inclusion as an essential element in the magnetic dynamo theory, which tries to explain the sustainement of magnetic fields on large scales over cosmic timescales (see Subramanian 2002; Brandenburg \& Subramanian 2005a,b). The possible operation of a large-scale dynamo for instance is directly connected to the generation of helicity in turbulent environments (see Shukurov et al. 2006; Brandenburg 2009; Sokoloff 2007), a process that, until now, could not be verified through observation.

This study is particularly concerned with the question of how to extract knowledge regarding turbulent magnetic helicity spectra from the statistical information found in radio-observational data involving polarization and Faraday rotation measurements. It was highly motivated by the studies of Volegova \& Stepanov (2010). 


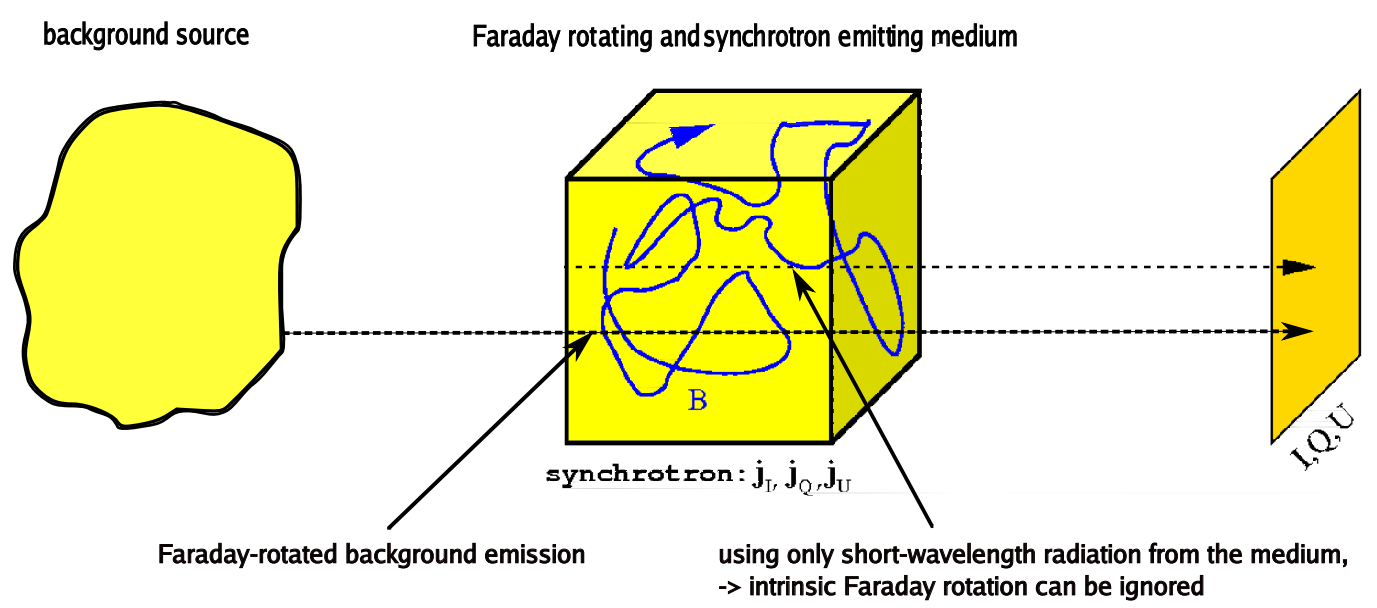

Fig. 1. Schematic of an observational situation for which our set of correlation functions is suitable (modified picture taken from Waelkens et al. 2009).

Information on magnetic fields can be imprinted onto radio data by two of the processes already mentioned above: Synchrotron emission and Faraday rotation. Nevertheless information is contained not only in their mean values, but also in higher order correlation and cross-correlation functions.

We therefore investigate a set of suitable radio observables for their cross-correlations, to see how these are connected to the statistical properties of the magnetic fields to be examined. This idea goes back to previous works by Spangler (1982, 1983), Eilek (1989a,b), Enßlin \& Vogt (2003), Kahniashvili \& Vachaspati (2006) and Waelkens et al. (2009). The set of radio observables we investigate contains total intensity $I(\boldsymbol{x})$, polarized intensity $P(\boldsymbol{x})$, and Faraday depth $\phi(\boldsymbol{x})$. We work out all correlation functions between them in a general framework. We restrict ourselves to fourth order in the magnetic field strength, and as far as possible, we do all calculations analytically. The aim is to find a direct relation to statistical properties of the magnetic fields, such as their power spectra.

The intensity $I(\boldsymbol{x})$ and the polarized intensity $P(\boldsymbol{x})$ are connected to the synchrotron emission within a magnetized volume. We assume them to be taken at sufficiently high frequencies thereby neglecting Faraday rotation on them. The Faraday depth $\phi(x)$ is measured via the Faraday rotation of a polarized background source at a different frequency seen through the same volume. The observational situation is visualized in Fig. 1.

With regard to these observable quantities, we can successfully establish all correlation functions in the form of analytical relations to the magnetic field power and helicity spectra while implementing Gaussian field statistics for simplicity. The result here should be to prove that it is possible to gain information about not only the total but also the helical part of the magnetic power spectrum, via $\left\langle P\left(\boldsymbol{k}_{\perp}\right) \phi\left(\boldsymbol{k}_{\perp}^{\prime}\right) \phi\left(\boldsymbol{k}_{\perp}^{\prime \prime}\right)\right\rangle_{\boldsymbol{B}}$ and $\left\langle I\left(\boldsymbol{k}_{\perp}\right) \phi\left(\boldsymbol{k}_{\perp}^{\prime}\right) \phi\left(\boldsymbol{k}_{\perp}^{\prime \prime}\right)\right\rangle_{\boldsymbol{B}}$.

Gaussian magnetic field statistics are not what numerical simulations of MHD turbulence find (see Waelkens et al. 2009); however, they are the starting point for any analysis of high-order correlation functions. In case all we know statistically about the fields is their two point correlation, the only assumption that solely expresses this knowledge is a Gaussian with the correlation tensor as the covariance matrix. Any other distribution function would contain more information in a Shannon-Boltzmann sense.

If additional information on higher order statistics is available, this could be incorporated by perturbative methods. These expand around the Gaussian case, which therefore has to be worked out first, as we do in this work.

Based on our results we further present the LITMUS test (Local Inference Test for Magnetic fields which Uncovers heliceS), a first simple procedure for probing data for helicity. An analysis of real and simulated data using this test, along with a thorough investigation of its applicability, can be found in Oppermann et al. (2010). The study is organized as follows. Section 2 presents our method and the general formalism we developed to evaluate the correlation functions analytically. Section 3 details a complete example calculation for one of the correlation functions, namely $\left\langle P\left(\boldsymbol{k}_{\perp}\right) \cdot \overline{P\left(\boldsymbol{k}_{\perp}^{\prime}\right)}\right\rangle_{B}$. Section 4 then presents all the correlation functions up to the fourth order in magnetic field strength. Section 5 introduces the LITMUS test. Section 6 finally presents a thorough evaluation of our findings. Details from the derivation of the other correlation functions are listed in the Appendix which also contains the remaining technical information regarding the study.

\section{Methods}

We now proceed to calculate the correlation functions of $I\left(\boldsymbol{x}_{\perp}\right), P\left(\boldsymbol{x}_{\perp}\right)$, and $\phi\left(\boldsymbol{x}_{\perp}\right)$. Since all calculations resemble each other in certain basic features, a general framework has been developed for them. Before presenting that, we introduce our basic notations and the magnetic correlation tensor, a quantity that will be referred to frequently but requires some preliminary explanation due to its complexity. 


\subsection{Notation}

We use the following definitions for the radio observables $I\left(\boldsymbol{x}_{\perp}\right), P\left(\boldsymbol{x}_{\perp}\right)$, and $\phi\left(\boldsymbol{x}_{\perp}\right)$ :

$I=\int \mathrm{d} z\left(B_{1}^{2}+B_{2}^{2}\right)$

$P=\int \mathrm{d} z\left(B_{1}^{2}-B_{2}^{2}+2 \mathrm{i} B_{1} B_{2}\right), \quad$ and

$\phi=\int \mathrm{d} z B_{3}$.

The coordinate axis $z$ always equals the line of sight. For convenience, all prefactors are suppressed including the electron density $n_{\mathrm{e}}$, assumed to be constant. A detailed derivation can be found in Appendix B.

Apart from this, we introduce some further notation we use frequently. In the following, the vectors $\boldsymbol{r}$ or $\boldsymbol{r}^{\prime}$ always denote a combination such as $\boldsymbol{x}^{\prime}-\boldsymbol{x}$ to be defined exactly when needed. Furthermore, $\boldsymbol{w}=\left(\boldsymbol{k}_{\perp}^{\prime \prime \prime}, 0\right), \boldsymbol{u}=\left(\boldsymbol{k}_{\perp}^{\prime \prime}, 0\right), \boldsymbol{v}=\left(\boldsymbol{k}_{\perp}^{\prime}, 0\right)$ and $\boldsymbol{a}=$ $\left(-\boldsymbol{q}_{\perp}-\boldsymbol{k}_{\perp}^{\prime},-q_{z}\right)$ holds.

\subsection{The magnetic correlation tensor}

We generally assume Gaussian statistics for the magnetic field distribution in the observed region. This is, of course, a simplification but represents an initial starting point, especially as we are mainly interested in a proof of concept, hence seek simplicity. In addition, we assume statistical homogeneity to first establish the most illuminating cases. This assumption is widely used in the literature. For an arbitrary field $\psi$, statistical homogeneity means that the two-point correlation function of the field depends only on the distance of the two parts, $\left\langle\psi\left(\boldsymbol{x}^{\prime}\right) \psi^{*}(\boldsymbol{x})\right\rangle=C(\boldsymbol{r})$ with $\boldsymbol{r}=\boldsymbol{x}^{\prime}-\boldsymbol{x}$. In Fourier space this automatically implies that

$\left\langle\psi\left(\boldsymbol{k}^{\prime}\right) \psi^{*}(\boldsymbol{k})\right\rangle=(2 \pi)^{3} \delta^{3}\left(\boldsymbol{k}^{\prime}-\boldsymbol{k}\right) P_{\psi}\left(k^{\prime}\right)$,

where the $\psi$-power spectrum is specified by the Fourier-transformed correlation function $P_{\psi}\left(k^{\prime}\right) \propto \int \mathrm{d} r^{3} C_{\psi}(r) \exp \left[\mathrm{i} k^{\prime} r\right]$ as stated by the Wiener-Kinchin-theorem (Bracewell 2000).

Within this study, the magnetic correlation tensor $M_{i j}\left(\boldsymbol{x}, \boldsymbol{x}^{\prime}\right)=\left\langle B_{i}(\boldsymbol{x}) B_{j}\left(\boldsymbol{x}^{\prime}\right)\right\rangle$ is frequently used for which this translational invariance leads to

$M_{i j}\left(\boldsymbol{x}, \boldsymbol{x}^{\prime}\right)=M_{i j}\left(\boldsymbol{x}^{\prime}-\boldsymbol{x}=\boldsymbol{r}\right) \quad$ in normal space, and

$\hat{M}_{i j}\left(\boldsymbol{k}, \boldsymbol{k}^{\prime}\right)=(2 \pi)^{3} \delta^{3}\left(\boldsymbol{k}^{\prime}-\boldsymbol{k}\right) \hat{M}_{i j}\left(\boldsymbol{k}^{\prime}\right)$ in Fourier space.

For homogeneous and isotropic magnetic turbulence, the translationally invariant magnetic correlation tensor can be written as

$M_{i j}(\boldsymbol{r})=M_{\mathrm{N}}(r) \delta_{i j}+\left(M_{\mathrm{L}}(r)-M_{\mathrm{N}}(r)\right) \frac{r_{i} r_{j}}{r^{2}}+M_{\mathrm{H}}(r) \epsilon_{i j m} r_{m}$

with the longitudinal, normal, and helical spectra denoted by $M_{\mathrm{L}}(r), M_{\mathrm{N}}(r)$, and $M_{\mathrm{H}}(r)$, respectively. The solenoidal condition $\nabla \cdot \boldsymbol{B}=0$ enables the connection of the two non-helical spectra by $M_{\mathrm{N}}(r)=\frac{1}{2 r} \frac{\mathrm{d}}{\mathrm{d} r}\left(r^{2} M_{\mathrm{L}}(r)\right)$. By applying a Fourier transformation, we obtain

$\hat{M}_{i j}(\boldsymbol{k})=\hat{M}_{\mathrm{N}}(k)\left(\delta_{i j}-\frac{k_{i} k_{j}}{k^{2}}\right)-\mathrm{i} \epsilon_{i j m} \hat{H}(k) \frac{k_{m}}{k}$.

In this case, the condition $\nabla \cdot \boldsymbol{B}=0$ was used directly in the form $k_{i} \hat{M}_{i j}(\boldsymbol{k})=0$ to reduce the degrees of freedom to the normal and the helical spectra. These two functions are specified in terms of their real space counterparts as

$\hat{M}_{\mathrm{N}}(k)=\int \mathrm{d} r^{3} M_{\mathrm{N}}(r) \exp [\mathrm{i} k r], \quad$ and

$\hat{H}(k)=\frac{\mathrm{d}}{\mathrm{d} k} \hat{M}_{\mathrm{H}}(k)=\frac{\mathrm{d}}{\mathrm{d} k} \int \mathrm{d} r^{3} M_{\mathrm{H}}(r) \exp [\mathrm{i} \boldsymbol{k} \boldsymbol{r}]$.

Some interesting properties are

$M_{\mathrm{N}}(0)=M_{\mathrm{L}}(0) \quad$ by definition,

$M_{i j}(0)=M_{\mathrm{N}}(0) \delta_{i j}, \quad$ and

$\hat{M}_{\mathrm{N}}(0)=0, \quad$ since $M_{\mathrm{N}}$ would diverge otherwise.

The magnetic correlation tensor is closely related to the energy spectrum of the magnetic field. The field's mean energy density can be expressed as

$\frac{1}{8 \pi}\left\langle\boldsymbol{B}^{2}(\boldsymbol{x})\right\rangle=\frac{1}{8 \pi} \int \frac{\mathrm{d} k^{3}}{(2 \pi)^{3}}\left\langle\sum_{i} B_{i}(\boldsymbol{k}) B_{i}(\boldsymbol{k})\right\rangle=\frac{1}{8 \pi} \int \frac{\mathrm{d} k^{3}}{(2 \pi)^{3}} \sum_{i} \hat{M}_{i i}(\boldsymbol{k})=\frac{1}{(2 \pi)^{3}} \int_{0}^{\infty} \mathrm{d} k k^{2} \hat{M}_{\mathrm{N}}(k) \stackrel{!}{=} \int_{0}^{\infty} \mathrm{d} k \epsilon_{B}(k)$. 
In this case, we have used $M_{i i}(k)=2 M_{\mathrm{N}}$ and $\epsilon_{B}(k)$ denotes the 1D-energy density of $\boldsymbol{B}$. From this we derive

$\epsilon_{B}(k)=\frac{k^{2} \hat{M}_{\mathrm{N}}(k)}{8 \pi^{3}}$,

which is used in the following to replace $M_{\mathrm{N}}(k)$ by the more commonly applied magnetic energy spectrum.

Analogously to (14), we can relate the helical part of the spectrum $\hat{H}(k)$ to the mean current helicity $\boldsymbol{j} \cdot \boldsymbol{B}$ and deduce a 1D-helical energy density $\epsilon_{\mathrm{H}}(k)$ :

$$
\begin{aligned}
\langle\boldsymbol{j} \cdot \boldsymbol{B}\rangle & =\langle\boldsymbol{B} \cdot(\nabla \times \boldsymbol{B})\rangle=\left.\left\langle B_{l}(\boldsymbol{r}) \epsilon_{l i j} \partial_{r_{i}} B_{j}(\boldsymbol{x}+\boldsymbol{r})\right\rangle\right|_{r=0}=\left.\partial_{r_{i}} \epsilon_{l i j} M_{l j}(r)\right|_{r=0} \\
& =\mathrm{i} \epsilon_{l i j} \int \frac{\mathrm{d} k^{3}}{(2 \pi)^{3}} k_{i} \hat{M}_{l j}(\boldsymbol{k})=\int \frac{\mathrm{d} k^{3}}{(2 \pi)^{3}} \epsilon_{l i j} \epsilon_{l j m} \hat{H}(k) \frac{k_{i} k_{m}}{k} \\
& =-\int \frac{\mathrm{d} k^{3}}{(2 \pi)^{3}} \underbrace{\epsilon_{l j i} \epsilon_{l j m}}_{2 \delta_{i m}} \hat{H}(k) \frac{k_{i} k_{m}}{k}=-\frac{8 \pi}{(2 \pi)^{3}} \int_{0}^{\infty} \mathrm{d} k k^{3} \hat{H}(k) \\
& \stackrel{!}{=} \int_{0}^{\infty} \mathrm{d} k \epsilon_{\mathrm{H}}(k) .
\end{aligned}
$$

We can read off the 1D-helical energy density $\epsilon_{\mathrm{H}}(k)$, which also can be used to substitute $H(k)$ :

$\epsilon_{\mathrm{H}}(k)=-\frac{k^{3} \hat{H}(k)}{\pi^{2}}$.

Nevertheless, in our calculations we want to relate to the helicity spectrum $\hat{R}(k)$ rather than to the current helicity spectrum $\hat{H}(k)$, since it is the helicity $\boldsymbol{B} \cdot \boldsymbol{A}$ that usually is the subject of magnetohydrodynamics and dynamo theory.

Fortunately, the current helicity and the magnetic helicity $\boldsymbol{B} \cdot \boldsymbol{A}$ are closely connected for isotropic, turbulent fields. Since the current helicity $(\nabla \times \boldsymbol{B}) \cdot \boldsymbol{B}$ has the same mathematical structure as the helicity $(\nabla \times \boldsymbol{A}) \cdot \boldsymbol{A}$, we can perform exactly the same derivation as in (16) to show that the mean helicity relates to the helicity spectrum $\hat{R}(k)$ of the correlation tensor for the vector potential in the same way as the mean current helicity relates to the current helicity spectrum of the magnetic correlation tensor. We can construct the correlation tensor $\left\langle\boldsymbol{A}(\boldsymbol{x}) \boldsymbol{A}^{*}\left(\boldsymbol{x}^{\prime}\right)\right\rangle$ for the magnetic vector potential $\boldsymbol{A}$ similar to the magnetic correlation tensor. If we assume translational invariance for the statistics and apply the Lorenz gauge condition $\nabla \cdot \boldsymbol{A}=0$ to the vector potential, all deductions made for the magnetic correlation tensor will also hold for the correlation tensor of the vector potential. In Fourier space it takes the form:

$\left\langle\boldsymbol{A}_{m}(\boldsymbol{k}) \boldsymbol{A}_{n}^{*}\left(\boldsymbol{k}^{\prime}\right)\right\rangle=\hat{R}_{m n}(\boldsymbol{k})=\hat{R}_{\mathrm{N}}(k)\left(\delta_{m n}-\frac{k_{m} k_{n}}{k^{2}}\right)-\mathrm{i} \epsilon_{m n v} \hat{R}_{\mathrm{H}}(k) \frac{k_{v}}{k}$.

From here we can start by rewriting the magnetic correlation tensor in terms of the correlation tensor for the vector potential:

$\left\langle\boldsymbol{B}_{i}(\boldsymbol{x}) \boldsymbol{B}_{j}^{*}\left(\boldsymbol{x}^{\prime}\right)\right\rangle=\epsilon_{i l m} \epsilon_{j r n} \partial_{\boldsymbol{x}_{l}} \partial_{\boldsymbol{x}_{r}}\left\langle\boldsymbol{A}_{m}(\boldsymbol{x}) \boldsymbol{A}_{n}^{*}\left(\boldsymbol{x}^{\prime}\right)\right\rangle$

If we now perfom a Fourier transformation on both sides and furthermore apply the condition for translational invariance (6) we get

$\int \frac{\mathrm{d} k^{3}}{(2 \pi)^{3}} \hat{M}_{i j}(\boldsymbol{k})=\int \frac{\mathrm{d} k^{3}}{(2 \pi)^{3}} \epsilon_{i l m} \epsilon_{j r n} k_{l} k_{r} \hat{R}_{m n}(\boldsymbol{k})$.

Thus, we find

$\hat{M}_{i j}(\boldsymbol{k})=\epsilon_{i l m} \epsilon_{j r n} k_{l} k_{r} \hat{R}_{m n}(\boldsymbol{k})$.

Using

$\epsilon_{i l m} \epsilon_{j r n} \epsilon_{m n v}=\left(\delta_{i v} \delta_{l n}-\delta_{i n} \delta_{l v}\right) \epsilon_{j r n}=\delta_{i v} \epsilon_{j r l}-\delta_{l v} \epsilon_{j r i}$

we can rewrite the righthand side of (21):

$\epsilon_{i l m} \epsilon_{j r n} k_{l} k_{r} \hat{R}_{m n}(\boldsymbol{k})=\underbrace{\epsilon_{i l m} \epsilon_{j r n} k_{l} k_{r}\left(\delta_{m n}-\frac{k_{m} k_{n}}{k^{2}}\right)}_{\text {symmetric in } i, j}-\underbrace{k_{i} \epsilon_{j r l} k_{r} k_{l} \hat{R}_{\mathrm{H}}(k) / k}_{=0}+\underbrace{i k^{2} \epsilon_{i j r} k_{r} \hat{R}_{\mathrm{H}}(k) / k}_{\text {antisymmetric in } i, j}$.

Finally, we find the relation between the current helicity spectrum $\hat{H}(k)$ and the helicity spectrum $\hat{R}_{\mathrm{H}}$ by identifying the antisymmetric part of the lefthand side of (21) with the antisymmetric part of the right hand side:

$\begin{aligned}-\mathrm{i} \epsilon_{i j m} k_{m} \hat{H}(k) / k & =+\mathrm{i} k^{2} \epsilon_{i j r} k_{r} \hat{R}_{\mathrm{H}}(k) / k \\ \Rightarrow \hat{H}(k) & =-k^{2} \hat{R}_{\mathrm{H}}(k) .\end{aligned}$ 
From now on, although we mostly speak of helicity, we are actually dealing with current helicity for convenience and bear in mind that $\hat{H}(k)$ is easily convertible to $\hat{R}_{\mathrm{H}}(k)$ using (24). A more detailed analysis of all these relations can be found in Moffatt (1978).

For the magnetic energy density in 1D Fourier space, a broken power law is assumed in the following in our examples by adopting

$\epsilon_{B}(k)=\epsilon_{0}\left(\frac{k}{k_{0}}\right)^{\beta}\left(1+\left(\frac{k}{k_{0}}\right)^{2}\right)^{-\frac{(\alpha+\beta)}{2}}$,

usually with $\beta=2$ and $k_{0}=1$ if not stated otherwise, but with different spectral indices $\alpha$. The low-k asymptotic $\epsilon_{B} \approx k^{2}$ corresponds to a white noise spectrum without correlations on scales larger than $1 / k_{0}$. For large $k$, we find $\epsilon_{B} \propto k^{-\alpha}$, eventually becoming a Kolmogorov-spectrum for $\alpha=5 / 3$.

Whenever necessary, we can always model the helicity power spectrum as $\hat{H}(k)=-\frac{\pi^{2}}{k^{3}} \epsilon_{\mathrm{H}}(k)=\frac{\pi^{2}}{k^{3}} h(k) \epsilon_{B}(k)$, where $h(k)$ is a function between -1 and 1 . This can be seen from

$\hat{M}_{i j}(\boldsymbol{k})=\frac{\epsilon_{B}(k)}{k^{2}} \underbrace{\left[8 \pi\left(\delta_{i j}-\frac{k_{i} k_{j}}{k^{2}}\right)-\mathrm{i} \pi^{2} \epsilon_{i j m} h(k) \frac{k_{m}}{k}\right]}_{A_{i j}}$

and from the fact that the matrix $A_{i j}$ must be positive definite. We adopt $\boldsymbol{k}=k e_{x}$ without loss of generality and find the characteristic polynomial of $A_{i j}$ to be

$$
\begin{aligned}
& (1-\lambda)^{2}-h^{2}=0 \\
& \longrightarrow 1 \pm h=\lambda \geq 0 \\
& \longrightarrow|h| \leq 1
\end{aligned}
$$

which yields $h \in[-1,1]$.

\subsection{General framework for all calculations}

The correlation functions of our observables can be calculated in a general and consistent way which we want to present now. Before we start, some general remarks about the mathematics are in order. Throughout this study an expression such as $\boldsymbol{J}^{\dagger} \boldsymbol{B}$ relates to a multidimensional scalar product:

$\boldsymbol{J}^{\dagger} \boldsymbol{B}=\sum_{i} \int \mathrm{d} x^{3} \boldsymbol{J}_{i}^{*}(x) \boldsymbol{B}_{i}(x)$.

This definition includes a discrete summation over indices, as well as a continuous integral over position space. The symmetric properties of matrix objects defined over a space with a scalar product (28) reflect the appearance of discrete summation and continous integration. Therefore, a matrix element $M_{i j}(\boldsymbol{x}, \boldsymbol{y})$ is called symmetric (or hermitian for complex quantities), if it is symmetric under a transposition of its indices and under an interchange of its vectors $r$ :

$M_{i j}^{\dagger}(\boldsymbol{x}, \boldsymbol{y})=M_{j i}(\boldsymbol{y}, \boldsymbol{x})$

Thus, a symmetrized element is expressed as

$M_{i j, \mathrm{sym}}(\boldsymbol{r})=\frac{1}{2}\left(M_{i j}(\boldsymbol{r})+M_{j i}(-\boldsymbol{r})\right)$

where $\boldsymbol{r}=\boldsymbol{y}-\boldsymbol{x}$. When a matrix element is only symmetrized for index transposition, we call it index-symmetric:

$M_{i j, \text { isym }}(\boldsymbol{x}, \boldsymbol{y})=\frac{1}{2}\left(M_{i j}(\boldsymbol{r})+M_{j i}(\boldsymbol{r})\right)$

This distinction between symmetric and index-symmetric is important, because it takes care of subtleties that could easily generate confusion. We would like to emphasize the difference between both symmetry operations, when applied to the magnetic correlation tensor (8). The tensor contains an intrinsic symmetric and an intrinsic antisymmetric element. Regarding (30), the intrinsic antisymmetric part is preserved, whereas for (31) it is not. This is of paramount importance, since information on the helical power spectrum is only preserved if the intrinsic antisymmetric parts do not cancel out during calculations. 
Furthermore, we introduce the functional derivative, which is the generalization of an ordinary derivative to functional vector spaces. Its precise definition is (see Peskin \& Schroeder 1995)

$$
\begin{aligned}
\frac{\delta}{\delta \boldsymbol{J}_{i}(\boldsymbol{x})} \boldsymbol{J}_{j}(\boldsymbol{y}) & =\delta^{3}(\boldsymbol{x}-\boldsymbol{y}) \delta_{i j} \\
\frac{\delta}{\delta \boldsymbol{J}_{i}(\boldsymbol{x})} \frac{1}{2} \boldsymbol{J}^{\dagger} \mathbf{M} \boldsymbol{J} & =\frac{\delta}{\delta \boldsymbol{J}_{i}(\boldsymbol{x})} \int \mathrm{d} y^{3} \int \mathrm{d} y^{\prime 3} \frac{1}{2} \boldsymbol{J}_{k}(\boldsymbol{y}) \mathbf{M}_{k l}\left(\boldsymbol{y}, \boldsymbol{y}^{\prime}\right) \boldsymbol{J}_{l}\left(\boldsymbol{y}^{\prime}\right) \\
& =\int \mathrm{d} y^{\prime 3}\left[\frac{1}{2} \mathbf{M}_{i l}\left(\boldsymbol{x}, \boldsymbol{y}^{\prime}\right) \boldsymbol{J}_{l}\left(\boldsymbol{y}^{\prime}\right)+\frac{1}{2} \boldsymbol{J}_{k}\left(\boldsymbol{y}^{\prime}\right) \mathbf{M}_{k i}\left(\boldsymbol{x}, \boldsymbol{y}^{\prime}\right)\right] \\
& =\frac{1}{2}\left(\boldsymbol{J}^{\dagger} \mathbf{M}\right)_{i}(\boldsymbol{x})+\frac{1}{2}(\mathbf{M} \boldsymbol{J})_{i}(\boldsymbol{x}) .
\end{aligned}
$$

For convenience and to avoid confusion with the delta function, we sometimes adopt an easier notation:

$\frac{\delta}{\delta \boldsymbol{J}_{i}(\boldsymbol{x})}=\partial_{J_{i}}(\boldsymbol{x})=\partial_{i}(\boldsymbol{x})$

Now we proceed, presenting the framework of the calculations. The general evaluation of the expectation value for a function $X$ of observables for Gaussian magnetic field statistics with covariance matrix $\mathbf{M}$ and its determinant $|\mathbf{M}|$ is conducted as follows ${ }^{1}$ :

$$
\begin{aligned}
\left\langle X\left(\boldsymbol{k}_{\perp}, \boldsymbol{k}_{\perp}^{\prime}, \ldots\right)\right\rangle_{\boldsymbol{B}}= & \frac{1}{\sqrt{\mid 2 \pi M} \mid} \int \mathcal{D} B X\left(\boldsymbol{k}_{\perp}, \boldsymbol{k}_{\perp}^{\prime}, \ldots\right) \exp \left[-\frac{1}{2} \boldsymbol{B}^{\dagger} \mathbf{M}^{-1} \boldsymbol{B}\right] \\
= & \frac{1}{\sqrt{\mid 2 \pi M}} \int \mathcal{D} B \int \mathrm{d} \boldsymbol{x}_{\perp} \ldots \int \mathrm{d} \boldsymbol{x}_{\perp}^{\prime \prime \prime} \int \mathrm{d} z \ldots \int \mathrm{d} z^{\prime \prime \prime} F\left(B_{i}(\boldsymbol{x}), B_{j}\left(\boldsymbol{x}^{\prime}\right), \ldots\right) \\
& \exp \left[-\frac{1}{2} \boldsymbol{B}^{\dagger} \mathbf{M}^{-1} \boldsymbol{B}\right] \exp \left[\mathrm{i}\left(\boldsymbol{k}_{\perp} \boldsymbol{x}_{\perp}+\boldsymbol{k}_{\perp}^{\prime} \boldsymbol{x}_{\perp}^{\prime}+\ldots\right)\right] \\
= & \left.\frac{1}{\sqrt{\mid 2 \pi M} \mid} \int \mathcal{D} B \int \mathrm{d} \boldsymbol{x}_{\perp} \ldots \int \mathrm{d} \boldsymbol{x}_{\perp}^{\prime \prime \prime} \int \mathrm{d} z \ldots \int \mathrm{d} z^{\prime \prime \prime} F\left(\partial_{J_{i}}(\boldsymbol{x}), \partial_{J_{j}}\left(\boldsymbol{x}^{\prime} \ldots\right)\right)\right|_{\boldsymbol{J}=0} \\
& \exp \left[-\frac{1}{2} \boldsymbol{B}^{\dagger} \mathbf{M}^{-1} \boldsymbol{B}+\boldsymbol{J}^{\dagger} \boldsymbol{B}\right] \exp \left[\mathrm{i}\left(\boldsymbol{k}_{\perp} \boldsymbol{x}_{\perp}+\boldsymbol{k}_{\perp}^{\prime} \boldsymbol{x}_{\perp}^{\prime}+\ldots\right)\right] \\
= & \int \mathrm{d} \boldsymbol{x}_{\perp} \ldots \int \mathrm{d} \boldsymbol{x}_{\perp}^{\prime \prime \prime} \int \mathrm{d} z \ldots \int \mathrm{d} z^{\prime \prime \prime} \exp \left[\mathrm{i}\left(\boldsymbol{k}_{\perp} \boldsymbol{x}_{\perp}+\boldsymbol{k}_{\perp}^{\prime} \boldsymbol{x}_{\perp}^{\prime}+\ldots\right)\right] \\
& F\left(\partial_{J_{i}}(\boldsymbol{x}),\left.\partial_{J_{j}}\left(\boldsymbol{x}^{\prime} \ldots\right)\right|_{\boldsymbol{J}=0} \exp \left[\frac{1}{2} \boldsymbol{J}^{\dagger} \mathbf{M} \boldsymbol{J}\right]\right. \\
= & \int \mathrm{d} \boldsymbol{x} \ldots \int \mathrm{d} \boldsymbol{x}^{\prime \prime \prime} \exp \left[\mathrm{i}\left(\boldsymbol{k}_{\perp} \boldsymbol{x}_{\perp}+\boldsymbol{k}_{\perp}^{\prime} \boldsymbol{x}_{\perp}^{\prime}+\ldots\right)\right] \\
& \left.F\left(\partial_{J_{i}}(\boldsymbol{x}), \partial_{J_{j}}\left(\boldsymbol{x}^{\prime} \ldots\right)\right)\right|_{\boldsymbol{J}=0} \exp \left[\frac{1}{2} \boldsymbol{J}^{\dagger} \mathbf{M} \boldsymbol{J}\right]
\end{aligned}
$$

At this point, it is necessary to consider the actual form of $F$. The calculations vary enormously as complexity of the correlation functions differs. From now on we identify the covariance matrix of the Gaussian distribution with the magnetic correlation tensor. This identity is proven in Appendix C. In (33) we introduced a generating functional $\boldsymbol{J}$ and completed the square of the exponential. By integrating out the shifted Gaussian part, which depends on $\boldsymbol{B}$, we are left with the part solely dependent on $\boldsymbol{J}$ :

$$
\begin{aligned}
\frac{1}{\sqrt{|2 \pi M|}} \int \mathcal{D} B \exp \left[-\frac{1}{2} \boldsymbol{B}^{\dagger} \mathbf{M}^{-1} \boldsymbol{B}+\boldsymbol{J}^{\dagger} \boldsymbol{B}\right] & =\frac{1}{\sqrt{\mid 2 \pi M}} \int \mathcal{D} B \exp \left[-\frac{1}{2} \boldsymbol{B}^{\dagger} \mathbf{M}^{-1} \boldsymbol{B}+\frac{1}{2} \boldsymbol{J}^{\dagger} \boldsymbol{B}+\frac{1}{2} \boldsymbol{B}^{\dagger} \boldsymbol{J}\right] \\
& =\frac{1}{\sqrt{\mid 2 \pi M}} \int \mathcal{D} B \exp \left[-\frac{1}{2} \boldsymbol{B}^{\dagger} \mathbf{M}^{-1} \boldsymbol{B}+\frac{1}{2} \boldsymbol{J}^{\dagger} \mathbf{M M}^{-1} \boldsymbol{B}+\frac{1}{2} \boldsymbol{B}^{\dagger} \mathbf{M}^{-1} \mathbf{M} \boldsymbol{J}\right] \\
& =\frac{1}{\sqrt{\mid 2 \pi M}} \int \mathcal{D} B \exp \left[-\frac{1}{2}(\boldsymbol{B}-\mathbf{M} \boldsymbol{J})^{\dagger} \mathbf{M}^{-1}(\boldsymbol{B}-\mathbf{M} \boldsymbol{J})\right] \exp \left[\frac{1}{2} \boldsymbol{J}^{\dagger} \mathbf{M} \boldsymbol{J}\right] \\
& =\exp \left[\frac{1}{2} \boldsymbol{J}^{\dagger} \mathbf{M} \boldsymbol{J}\right] .
\end{aligned}
$$

In (34), we finally made the assumption that the observed space is sufficiently extended, so that we can neglect the finiteness of the integrals over the lines of sight $\int \mathrm{d} z$ and treat them as if they were infinite.

\footnotetext{
${ }^{1}$ We denote with $X$ either $I, P$, or $\phi$ or combinations thereof up to the fourth order in the magnetic field in Fourier space. Therefore $X$ contains up to 4 Fourier vectors within the observed plane labeled with primes. We denote with $F$ the real space source function of $X$ that depends directly on the local components of the magnetic field $B_{i}$ so that $X=\int \mathrm{d} z \ldots \int \mathrm{d} z^{\prime \prime \prime} F\left(\boldsymbol{B}, \boldsymbol{B}^{\prime}, \boldsymbol{B}^{\prime \prime}, \boldsymbol{B}^{\prime \prime \prime}\right)$.
} 
Introducing the generating functional $\boldsymbol{J}$, thereby changing the fields $\boldsymbol{B}(\boldsymbol{x})$ to the functional derivative $\partial_{\boldsymbol{J}_{i}}(\boldsymbol{x})=\frac{\partial}{\partial \boldsymbol{J}_{i}}(\boldsymbol{x})$ in $(33)$ provides a powerful method for calculating the integral $\mathcal{D} B$ over all possible magnetic field configurations as an infinite-dimensional path integral. With the definition of the functional derivative at hand (32), we can discuss its actual evaluation. Since all uneven products of functional derivatives in (34) give zero because they also leave expressions with $\boldsymbol{J}$ that have been "taken down" from the exponential function during the differentiation, we are left with just two general types of possible combinations: two or four derivatives.

In addition to the generating functional technique familiar from quantum field theory, we also apply the renowned Wick theorem (see for example Peskin \& Schroeder 1995) to evaluate the remaining derivatives in an elegant, quick, and safe manner, rather than calculating them by brute force. The Wick theorem can be used under conditions that will become clear if one looks at how the underlying differentiation works. Most importantly, we need the covariance matrix to be symmetric or hermitian. This, as already mentioned, means

$M_{i j}(\boldsymbol{x}, \boldsymbol{y})=M_{i j}^{\dagger}(\boldsymbol{x}, \boldsymbol{y})=M_{j i}(\boldsymbol{y}, \boldsymbol{x})$

which is fulfilled by (7) and (8). However, since there will be one case where the covariance matrix is not symmetric or hermitian (see Sect. 3) and since we have to pay full attention to the exact order of the vectors, discussed in more detail below, the Wick theorem is expressed in a form that keeps track of these subtleties:

$$
\begin{aligned}
\left.\partial_{i}(\boldsymbol{a}) \partial_{j}(\boldsymbol{b}) \exp \left[\frac{1}{2} \boldsymbol{J}^{\dagger} \mathbf{M} \boldsymbol{J}\right]\right|_{\boldsymbol{J}=0} & =\left.\partial_{i}(\boldsymbol{a})\left[\frac{1}{2}\left(\boldsymbol{J}^{\dagger} \mathbf{M}\right)_{j}(\boldsymbol{b})+\frac{1}{2}(\mathbf{M} \boldsymbol{J})_{j}(\boldsymbol{b})\right] \exp \left[\frac{1}{2} \boldsymbol{J}^{\dagger} \mathbf{M} \boldsymbol{J}\right]\right|_{\boldsymbol{J}=0} \\
& =\frac{1}{2} \mathbf{M}_{i j}(\boldsymbol{a}, \boldsymbol{b})+\frac{1}{2} \mathbf{M}_{j i}(\boldsymbol{b}, \boldsymbol{a})
\end{aligned}
$$

and

$$
\begin{aligned}
& \left.\partial_{i}(\boldsymbol{a}) \partial_{j}(\boldsymbol{b}) \partial_{k}(\boldsymbol{c}) \partial_{l}(\boldsymbol{d}) \exp \left[\frac{1}{2} \boldsymbol{J}^{\dagger} \mathbf{M} \boldsymbol{J}\right]\right|_{\boldsymbol{J}=0}=\left.\partial_{i}(\boldsymbol{a}) \partial_{j}(\boldsymbol{b}) \partial_{k}(\boldsymbol{c})\left[\frac{1}{2}\left(\boldsymbol{J}^{\dagger} \mathbf{M}\right)_{l}(\boldsymbol{d})+\frac{1}{2}(\mathbf{M} \boldsymbol{J}){ }_{l}(\boldsymbol{d})\right] \exp \left[\frac{1}{2} \boldsymbol{J}^{\dagger} \mathbf{M} \boldsymbol{J}\right]\right|_{\boldsymbol{J}=0} \\
& =\partial_{i}(\boldsymbol{a}) \partial_{j}(\boldsymbol{b})\left[\left(\frac{1}{2} \mathbf{M}_{k l}(\boldsymbol{c}, \boldsymbol{d})+\frac{1}{2} \mathbf{M}_{l k}(\boldsymbol{d}, \boldsymbol{c})\right)\right. \\
& \left.+\left(\frac{1}{2}(\mathbf{M} \boldsymbol{J})_{l}(\boldsymbol{d})+\frac{1}{2}\left(\boldsymbol{J}^{\dagger} \mathbf{M}\right)_{l}(\boldsymbol{d})\right)\left(\frac{1}{2}\left(\boldsymbol{J}^{\dagger} \mathbf{M}\right)_{k}(\boldsymbol{c})+\frac{1}{2}(\mathbf{M} \boldsymbol{J})_{k}(\boldsymbol{c})\right)\right]\left.\exp \left[\frac{1}{2} \boldsymbol{J}^{\dagger} \mathbf{M} \boldsymbol{J}\right]\right|_{\boldsymbol{J}=0} \\
& =\partial_{i}(\boldsymbol{a})\left[\left(\frac{1}{2} \mathbf{M}_{k l}(\boldsymbol{c}, \boldsymbol{d})+\frac{1}{2} \mathbf{M}_{l k}(\boldsymbol{d}, \boldsymbol{c})\right)\left(\frac{1}{2}\left(\boldsymbol{J}^{\dagger} \mathbf{M}\right)_{j}(\boldsymbol{b})+\frac{1}{2}(\mathbf{M} \boldsymbol{J})_{j}(\boldsymbol{b})\right)\right. \\
& +\left(\frac{1}{2} \mathbf{M}_{l j}(\boldsymbol{d}, \boldsymbol{b})+\frac{1}{2} \mathbf{M}_{j l}(\boldsymbol{b}, \boldsymbol{d})\right)\left(\frac{1}{2}\left(\boldsymbol{J}^{\dagger} \mathbf{M}\right)_{j}(\boldsymbol{c})+\frac{1}{2}(\mathbf{M} \boldsymbol{J})_{j}(\boldsymbol{c})\right) \\
& +\left.\left(\frac{1}{2}\left(\boldsymbol{J}^{\dagger} \mathbf{M}\right)_{l}(\boldsymbol{d})+\frac{1}{2}(\mathbf{M} \boldsymbol{J})_{l}(\boldsymbol{d})\right)\left(\frac{1}{2} \mathbf{M}_{k j}(\boldsymbol{c}, \boldsymbol{b})+\frac{1}{2} \mathbf{M}_{j k}(\boldsymbol{b}, \boldsymbol{c})+\ldots\right] \exp \left[\frac{1}{2} \boldsymbol{J}^{\dagger} \mathbf{M} \boldsymbol{J}\right]\right|_{\boldsymbol{J}=0} \\
& =\left(\frac{1}{2} M_{k l}(\boldsymbol{c}, \boldsymbol{d})+\frac{1}{2} M_{l k}(\boldsymbol{d}, \boldsymbol{c})\right)\left(\frac{1}{2} M_{i j}(\mathbf{a}, \boldsymbol{b})+\frac{1}{2} M_{j i}(\boldsymbol{b}, \mathbf{a})\right) \\
& +\left(\frac{1}{2} M_{l j}(\boldsymbol{d}, \boldsymbol{b})+\frac{1}{2} M_{j l}(\boldsymbol{b}, \boldsymbol{d})\right)\left(\frac{1}{2} M_{k i}(\boldsymbol{c}, \mathbf{a})+\frac{1}{2} M_{i k}(\mathbf{a}, \boldsymbol{c})\right) \\
& +\left(\frac{1}{2} M_{i l}(\mathbf{a}, \boldsymbol{d})+\frac{1}{2} M_{l i}(\boldsymbol{d}, \mathbf{a})\right)\left(\frac{1}{2} M_{k j}(\boldsymbol{c}, \boldsymbol{b})+\frac{1}{2} M_{j k}(\boldsymbol{b}, \boldsymbol{c})\right) .
\end{aligned}
$$

Now all derivatives up to fourth order can be calculated by simply inserting the current case. For example, $\left.\partial_{1}^{2}(\boldsymbol{x}) \partial_{1}^{2}\left(\boldsymbol{x}^{\prime}\right)\right|_{\boldsymbol{J}=0} \exp \left[\frac{1}{2} \boldsymbol{J}^{\dagger} \mathbf{M} \boldsymbol{J}\right]$ can be read from (38) by inserting $i=j=k=l=1, \boldsymbol{a}=\boldsymbol{b}=\boldsymbol{x}$ and $\boldsymbol{c}=\boldsymbol{d}=\boldsymbol{x}^{\prime}$ :

$\left.\partial_{1}^{2}(\boldsymbol{x}) \partial_{1}^{2}\left(\boldsymbol{x}^{\prime}\right) \exp \left[\frac{1}{2} \boldsymbol{J}^{\dagger} \mathbf{M} \boldsymbol{J}\right]\right|_{\boldsymbol{J}=0}=M_{11}^{2}(0)+2 M_{11}^{2}(\boldsymbol{r})$.

As we can see from (38), all antisymmetric parts cancel out during the differentiation. Thus although $\mathbf{M}$ was not explicitly restricted to be symmetric, only the symmetric elements of the magnetic correlation tensor

$M_{i j, \mathrm{sym}}(\boldsymbol{r})=\frac{1}{2}\left(M_{i j}(\boldsymbol{r})+M_{j i}(-\boldsymbol{r})\right)$

remain in the end. However, it is important to understand that these symmetric elements actually preserve the intrinsic antisymmetric parts that constitute the magnetic correlation tensor. As mentioned above, this is because we take the inversion of the vector $\boldsymbol{r}$ into account when transposing the tensor elements. A look at (7) reveals, that the minus sign of the Levi-Civita-tensor $\epsilon_{i j m}$ we encounter under interchanged indices is cancelled exactly by the minus sign occurring because of the inversion of the vector $\boldsymbol{r}$ :

$M_{i j}(\boldsymbol{r})=M_{\mathrm{N}}(r) \delta_{i j}+\left(M_{\mathrm{L}}(r)-M_{\mathrm{N}}(r)\right) \frac{r_{i} r_{j}}{r^{2}}+M_{\mathrm{H}}(r) \epsilon_{i j m} r_{m}$ 
This means that, although the tensor (41) is symmetric in the general way defined in (30), it is not index-symmetric due to its individual antisymmetric constituents. The consequence is that, if we carry out the derivatives using the Wick theorem, we not only have to keep track for the right combination of indices but also the corresponding vectors $\boldsymbol{r}$ or $-\boldsymbol{r}$ and, in the end only the symmetric parts of $\mathbf{M}$, as defined in (30), appear. And furthermore, this means that, if we encounter index-symmetric expressions such as $M_{i j}(\boldsymbol{r})=\left(M_{i j}(\boldsymbol{r})+M_{j i}(\boldsymbol{r})\right) / 2$, the intrinsic antisymmetric part related to the helical power spectrum in (41) is lost during the differentiation. A careful look at (38) reveals that with the right combination for $i, j, k, l$ and $\boldsymbol{a}, \boldsymbol{b}, \boldsymbol{c}, \boldsymbol{d}$ and a sum of terms as in (38), it is possible to get these combinations; for example,

$$
\begin{aligned}
& \left.\left(\partial_{1}^{2}(\boldsymbol{x}) \partial_{2}^{2}\left(\boldsymbol{x}^{\prime}\right)+\partial_{2}^{2}(\boldsymbol{x}) \partial_{1}^{2}\left(\boldsymbol{x}^{\prime}\right)\right) \exp \left[\frac{1}{2} \boldsymbol{J}^{\dagger} \mathbf{M} \boldsymbol{J}\right]\right|_{\boldsymbol{J}=0}=2 M_{11}(0) M_{22}(0) \\
& \quad+2\left(M_{21}^{2}\left(\boldsymbol{x}-\boldsymbol{x}^{\prime}\right)+M_{12}^{2}\left(\boldsymbol{x}-\boldsymbol{x}^{\prime}\right)\right)=2 M_{11}(0) M_{22}(0)+4 M_{21, \text { isym }}^{2}\left(\boldsymbol{x}-\boldsymbol{x}^{\prime}\right)
\end{aligned}
$$

\section{The polarization 2-point function $\left\langle P\left(k_{\perp}\right) \cdot P^{*}\left(k_{\perp}^{\prime}\right)\right\rangle_{B}$}

In this section the polarization 2-point function $\left\langle P\left(\boldsymbol{k}_{\perp}\right) \cdot P^{*}\left(\boldsymbol{k}_{\perp}^{\prime}\right)\right\rangle_{B}$ is calculated to serve us as an example for the general calculation to obtain the other correlation functions of our observables. Since the steps are similar for all correlation functions and differ only in complexity, we intend to present them in detail only for a single case here and just list the other calculations in the Appendix A.

As $\left\langle P\left(\boldsymbol{k}_{\perp}\right) \cdot P^{*}\left(\boldsymbol{k}_{\perp}^{\prime}\right)\right\rangle_{B}$ is of fourth order in the magnetic field and, in addition, $P$ has a rather complex dependence on $\boldsymbol{B}$, it is convenient to introduce a compact notation for $P\left(\boldsymbol{x}_{\perp}\right)=\int_{0}^{L} \mathrm{~d} z\left(B_{1}(\boldsymbol{x})+\mathrm{i} B_{2}(\boldsymbol{x})\right)^{2}$ in order to clarify the calculation as far as possible. Defining $B_{ \pm}=\frac{1}{\sqrt{2}}\left(B_{1} \pm \mathrm{i} B_{2}\right)$ allows the expression $P\left(\boldsymbol{x}_{\perp}\right)=\int_{0}^{L} \mathrm{~d} z 2 B_{+}^{2}(\boldsymbol{x})$ and $P^{*}\left(\boldsymbol{x}_{\perp}\right)=\int_{0}^{L} \mathrm{~d} z 2 B_{-}^{2}(\boldsymbol{x})$. Thus, a change in basis of $\boldsymbol{B}$ is introduced, mapping $\boldsymbol{B}=\left(B_{1}, B_{2}, B_{3}\right) \longrightarrow \tilde{\boldsymbol{B}}=\left(B_{+}, B_{-}, B_{3}\right)$. We can then work effectively with $2 B_{+}^{2}(\boldsymbol{x}) \cdot 2 B_{-}^{2}\left(\boldsymbol{x}^{\prime}\right)$ instead of $\left(B_{1}(\boldsymbol{x})+\mathrm{i} B_{2}(\boldsymbol{x})\right)^{2} \cdot\left(B_{1}\left(\boldsymbol{x}^{\prime}\right)-\mathrm{i} B_{2}\left(\boldsymbol{x}^{\prime}\right)\right)^{2}$. With regard to the correlation function, this results in

$$
\begin{aligned}
\left\langle P\left(\boldsymbol{k}_{\perp}\right) \cdot P^{*}\left(\boldsymbol{k}_{\perp}^{\prime}\right)\right\rangle_{B}= & 4 \int \mathrm{d} \boldsymbol{x}_{\perp} \int \mathrm{d} \boldsymbol{x}_{\perp}^{\prime} \int \mathrm{d} z \int \mathrm{d} z^{\prime} \exp \left[\mathrm{i}\left(\boldsymbol{k}_{\perp} \boldsymbol{x}_{\perp}-\boldsymbol{k}_{\perp}^{\prime} \boldsymbol{x}_{\perp}^{\prime}\right)\right] \\
& \left.\partial_{J_{+}}^{2}(\boldsymbol{x}) \partial_{J_{-}}^{2}\left(\boldsymbol{x}^{\prime}\right) \exp \left[\frac{1}{2} \boldsymbol{J}^{\dagger} \mathbf{M} \boldsymbol{J}\right]\right|_{\boldsymbol{J}=0} \cdot
\end{aligned}
$$

Regarding the differentiation with respect to $J_{ \pm}$we need to establish a relation between $J_{1 / 2}$ and $J_{ \pm}$. The basis-transformation should preserve all scalar products; therefore, we have $J_{+}^{\dagger} B_{+}+J_{-}^{\dagger} B_{-}+J_{3}^{\dagger} B_{3}=J_{1}^{\dagger} B_{1}+J_{2}^{\dagger} B_{2}+J_{3}^{\dagger} B_{3}$. Using this we establish the required relations:

$J_{+}^{+} B_{+}+J_{-}^{\dagger} B_{-}=J_{1}^{\dagger} B_{1}+J_{2}^{\dagger} B_{2}$

$\longrightarrow J_{1}^{\dagger}=\frac{1}{\sqrt{2}}\left(J_{+}^{\dagger}+J_{-}^{\dagger}\right)$ and $J_{2}^{\dagger}=\frac{\mathrm{i}}{\sqrt{2}}\left(J_{+}^{\dagger}-J_{-}^{\dagger}\right)$

from which we obtain $J_{ \pm}=\frac{1}{\sqrt{2}}\left(J_{1} \pm \mathrm{i} J_{2}\right)$

and also $\quad J_{1}=\frac{1}{\sqrt{2}}\left(J_{-}+J_{+}\right)$as well as $J_{2}=\frac{\mathrm{i}}{\sqrt{2}}\left(J_{-}-J_{+}\right)$.

Thus, the transformation matrices $\boldsymbol{J}=\mathbf{O} \tilde{\boldsymbol{J}}$ and $\tilde{\boldsymbol{J}}=\mathbf{O}^{\dagger} \boldsymbol{J}$ are

$\mathbf{O}=\left(\begin{array}{ccc}\frac{1}{\sqrt{2}} & \frac{1}{\sqrt{2}} & 0 \\ -\frac{i}{\sqrt{2}} & \frac{i}{\sqrt{2}} & 0 \\ 0 & 0 & 1\end{array}\right), \quad$ and $\mathbf{O}^{\dagger}=\left(\begin{array}{ccc}\frac{1}{\sqrt{2}} & \frac{i}{\sqrt{2}} & 0 \\ \frac{1}{\sqrt{2}} & -\frac{i}{\sqrt{2}} & 0 \\ 0 & 0 & 1\end{array}\right)$

We now need to express the argument $\boldsymbol{J}^{\dagger} \mathbf{M} \boldsymbol{J}$ of the exponential in (43) in terms of the transformed quantities

$\boldsymbol{J}^{\dagger} \mathbf{M} \boldsymbol{J}=\tilde{\boldsymbol{J}}^{\dagger} \mathbf{O}^{\dagger} \mathbf{M O} \tilde{\boldsymbol{J}}=\tilde{\boldsymbol{J}}^{\dagger} \tilde{\mathbf{M}} \tilde{\boldsymbol{J}} \quad$ with $\quad \tilde{\mathbf{M}}=\mathbf{O}^{\dagger} \mathbf{M O}$ 
Some elements of $\tilde{\mathbf{M}}$ that will soon become important are

$$
\begin{aligned}
& \tilde{M}_{++}(r)=\frac{1}{2}\left(M_{11}(r)+M_{22}(r)-\mathrm{i} M_{12}(r)+\mathrm{i} M_{21}(r)\right) \\
& \tilde{M}_{--}(r)=\frac{1}{2}\left(M_{11}(r)+M_{22}(r)+\mathrm{i} M_{12}(r)-\mathrm{i} M_{21}(r)\right) \\
& \tilde{M}_{+-}(r)=\frac{1}{2}\left(M_{11}(r)-M_{22}(r)+\mathrm{i} M_{12}(r)+\mathrm{i} M_{21}(r)\right) \\
& \tilde{M}_{-+}(r)=\frac{1}{2}\left(M_{11}(r)-M_{22}(r)-\mathrm{i} M_{12}(r)-\mathrm{i} M_{21}(r)\right) .
\end{aligned}
$$

The entire matrix then reads as

$$
\tilde{\mathbf{M}}=\left(\begin{array}{ccc}
\tilde{M}_{++}(r) & \tilde{M}_{+-}(r) & \frac{1}{\sqrt{2}}\left(M_{13}+M_{23}\right) \\
\tilde{M}_{-+}(r) & \tilde{M}_{--}(r) & \frac{1}{\sqrt{2}}\left(M_{13}-M_{23}\right) \\
\frac{1}{\sqrt{2}}\left(M_{31}-\mathrm{i} M_{32}\right) & \frac{1}{\sqrt{2}}\left(M_{31}+\mathrm{i} M_{32}\right) & M_{33}
\end{array}\right) .
$$

Returning to the correlation function (43), we can now carry out the functional derivatives. Since $\tilde{\boldsymbol{J}}$ is a complex quantity, we now have to be concerned with the complex conjugation implied in the $\dagger$ operation, which affects $\tilde{\boldsymbol{J}}^{*}=\left(J_{+}^{*}, J_{-}^{*}, J_{3}^{*}\right)=\left(J_{-}, J_{+}, J_{3}\right)$. Using (38) we find

$\left.\partial_{J_{+}}(\boldsymbol{x}) \partial_{J_{+}}(\boldsymbol{x}) \partial_{J_{-}}\left(\boldsymbol{x}^{\prime}\right) \partial_{J_{-}}\left(\boldsymbol{x}^{\prime}\right) \exp \left[\frac{1}{2} \tilde{\boldsymbol{J}}^{\dagger} \tilde{\mathbf{M}} \tilde{\boldsymbol{J}}\right]\right|_{\tilde{J}=0}=2\left(M_{11}(\boldsymbol{r})+M_{22}(\boldsymbol{r})\right)^{2}$

Inserting this into the overall equation for the correlation function (43) results in

$$
\begin{aligned}
& \left\langle P\left(\boldsymbol{k}_{\perp}\right) \cdot P^{*}\left(\boldsymbol{k}_{\perp}^{\prime}\right)\right\rangle=8 \int \mathrm{d} x^{3} \int \mathrm{d} x^{3^{\prime}}\left(M_{11}(\boldsymbol{r})+M_{22}(\boldsymbol{r})\right)^{2} \exp \left[\mathrm{i}\left(\boldsymbol{k}_{\perp} \boldsymbol{x}_{\perp}-\boldsymbol{k}_{\perp}^{\prime} \boldsymbol{x}_{\perp}^{\prime}\right)\right] \\
& =8 \int \mathrm{d} x^{3} \int \mathrm{d} r^{3} \cdot\left(M_{11}^{2}(\boldsymbol{r})+M_{22}^{2}(\boldsymbol{r})+2 M_{22}(\boldsymbol{r}) M_{11}(\boldsymbol{r})\right) \exp \left[\mathrm{i} \boldsymbol{x}_{\perp}\left(\boldsymbol{k}_{\perp}-\boldsymbol{k}_{\perp}^{\prime}\right)\right] \\
& \exp \left[-\mathrm{i} \boldsymbol{r}_{\perp} \boldsymbol{k}_{\perp}^{\prime}\right] \\
& =8 \int \mathrm{d} x^{3} \int \mathrm{d} r^{3} \int \frac{\mathrm{d} q^{3}}{(2 \pi)^{3}} \int \frac{\mathrm{d} q^{3}}{(2 \pi)^{3}} \cdot\left(\hat{M}_{11}(\boldsymbol{q}) \hat{M}_{11}\left(\boldsymbol{q}^{\prime}\right)+\hat{M}_{22}(\boldsymbol{q}) \hat{M}_{22}\left(\boldsymbol{q}^{\prime}\right)\right. \\
& \left.+\hat{M}_{22}(\boldsymbol{q}) \hat{M}_{11}\left(\boldsymbol{q}^{\prime}\right)+\hat{M}_{22}\left(\boldsymbol{q}^{\prime}\right) \hat{M}_{11}(\boldsymbol{q})\right) \exp \left[\mathrm{i} \boldsymbol{x}_{\perp}\left(\boldsymbol{k}_{\perp}-\boldsymbol{k}_{\perp}^{\prime}\right)\right] \exp \left[-\mathrm{i} \boldsymbol{r}\left(\boldsymbol{q}+\boldsymbol{q}^{\prime}\right)\right] \\
& \exp \left[-\mathrm{i} \boldsymbol{r}_{\perp} \boldsymbol{k}_{\perp}^{\prime}\right] \\
& =8(2 \pi)^{2} \delta^{2}\left(\boldsymbol{k}_{\perp}-\boldsymbol{k}_{\perp}^{\prime}\right) \int \mathrm{d} z \int \mathrm{d} r^{3} \int \frac{\mathrm{d} q^{3}}{(2 \pi)^{3}} \int \frac{\mathrm{d} q^{\prime 3}}{(2 \pi)^{3}} \cdot\left(\hat{M}_{11}(\boldsymbol{q}) \hat{M}_{11}\left(\boldsymbol{q}^{\prime}\right)\right. \\
& \left.+\hat{M}_{22}(\boldsymbol{q}) \hat{M}_{22}\left(\boldsymbol{q}^{\prime}\right)+\hat{M}_{22}(\boldsymbol{q}) \hat{M}_{11}\left(\boldsymbol{q}^{\prime}\right)+\hat{M}_{22}\left(\boldsymbol{q}^{\prime}\right) \hat{M}_{11}(\boldsymbol{q})\right) \exp \left[-\mathrm{i} \boldsymbol{r}_{\perp}\left(\boldsymbol{q}_{\perp}+\boldsymbol{q}_{\perp}^{\prime}+\boldsymbol{k}_{\perp}^{\prime}\right)\right] \\
& \exp \left[-\mathrm{i} r_{z}\left(q_{z}+q_{z}^{\prime}\right)\right] \\
& =\frac{8}{2 \pi} \delta^{2}\left(\boldsymbol{k}_{\perp}-\boldsymbol{k}_{\perp}^{\prime}\right) L_{z} \int \mathrm{d} q^{3} \int \mathrm{d} q^{\prime 3} \cdot \delta^{2}\left(\boldsymbol{q}_{\perp}+\boldsymbol{q}_{\perp}^{\prime}+\boldsymbol{k}_{\perp}^{\prime}\right) \delta\left(q_{z}+q_{z}^{\prime}\right)\left(\hat{M}_{11}(\boldsymbol{q}) \hat{M}_{11}\left(\boldsymbol{q}^{\prime}\right)\right. \\
& \left.+\hat{M}_{22}(\boldsymbol{q}) \hat{M}_{22}\left(\boldsymbol{q}^{\prime}\right)+\hat{M}_{22}(\boldsymbol{q}) \hat{M}_{11}\left(\boldsymbol{q}^{\prime}\right)+\hat{M}_{22}\left(\boldsymbol{q}^{\prime}\right) \hat{M}_{11}(\boldsymbol{q})\right) \\
& \stackrel{\boldsymbol{a}=\left(-\boldsymbol{q}_{\perp}-\boldsymbol{k}_{\perp}^{\prime},-q_{z}\right)}{=} \frac{8}{2 \pi} \delta^{2}\left(\boldsymbol{k}_{\perp}-\boldsymbol{k}_{\perp}^{\prime}\right) L_{z} \int \mathrm{d} q^{3} \cdot\left(\hat{M}_{11}(\boldsymbol{q}) \hat{M}_{11}(\boldsymbol{a})+\hat{M}_{22}(\boldsymbol{q}) \hat{M}_{22}(\boldsymbol{a})\right. \\
& \left.+\hat{M}_{22}(\boldsymbol{q}) \hat{M}_{11}(\boldsymbol{a})+\hat{M}_{22}(\boldsymbol{a}) \hat{M}_{11}(\boldsymbol{q})\right) \\
& =\frac{8}{2 \pi}(2 \pi)^{6} \delta^{2}\left(\boldsymbol{k}_{\perp}-\boldsymbol{k}_{\perp}^{\prime}\right) L_{z} \int \mathrm{d} q^{3} \cdot \frac{\epsilon_{B}(q) \epsilon_{B}(a)}{q^{2} a^{2}} \cdot\left[\left(1-\frac{q_{x}^{2}}{q^{2}}\right)\left(1-\frac{a_{x}^{2}}{a^{2}}\right)\right. \\
& \left.+\left(1-\frac{q_{y}^{2}}{q^{2}}\right)\left(1-\frac{a_{y}^{2}}{a^{2}}\right)+\left(1-\frac{q_{y}^{2}}{q^{2}}\right)\left(1-\frac{a_{x}^{2}}{a^{2}}\right)+\left(1-\frac{q_{x}^{2}}{q^{2}}\right)\left(1-\frac{a_{y}^{2}}{a^{2}}\right)\right] \\
& =8(2 \pi)^{5} \delta^{2}\left(\boldsymbol{k}_{\perp}-\boldsymbol{k}_{\perp}^{\prime}\right) L_{z} \underbrace{\int \mathrm{d} q^{3} \cdot \frac{\epsilon_{B}(q) \epsilon_{B}(a)}{q^{2} a^{2}}\left[\left(2-\frac{q_{\perp}^{2}}{q^{2}}\right)\left(2-\frac{a_{\perp}^{2}}{a^{2}}\right)\right]}_{K} .
\end{aligned}
$$

This integral can be further simplified. To do this, we transform it into spherical coordinates and perform the subintegral over $\varphi$ analytically. The remaining 2D integral can be done numerically without problems. We choose the axes to be selected so that the 

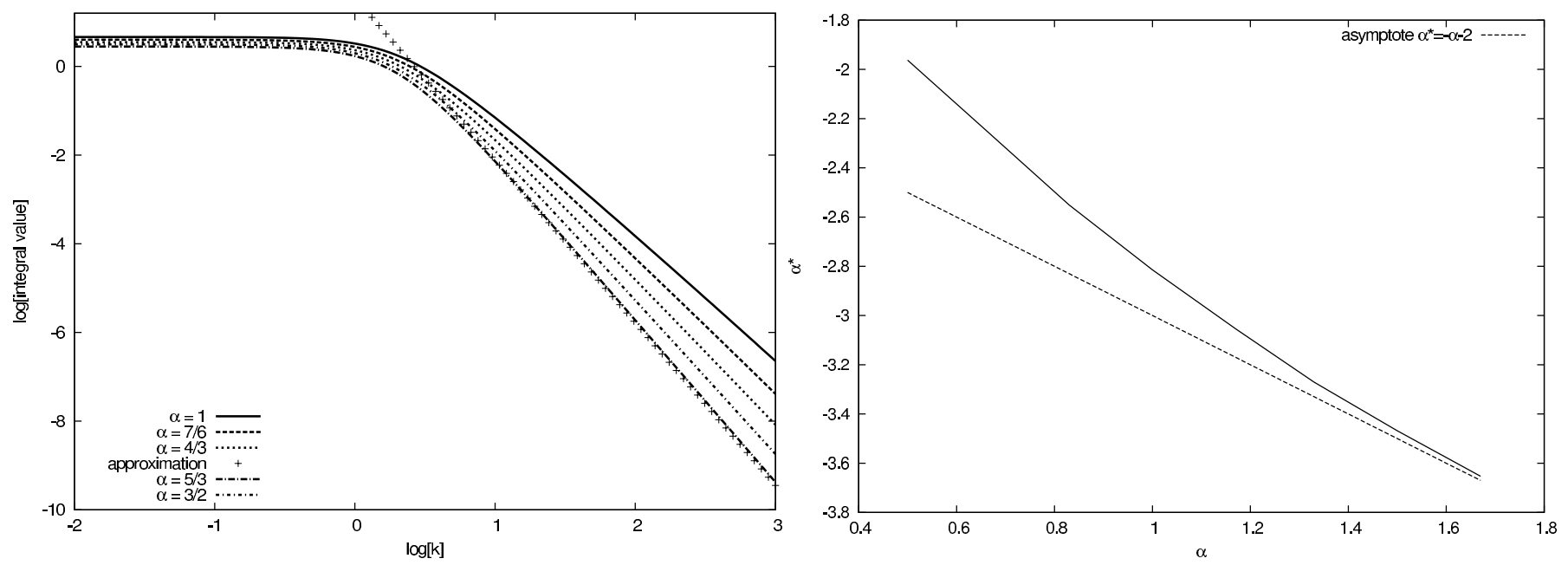

Fig. 2. Left: numerically evaluated integral values of $\left\langle P\left(\boldsymbol{k}_{\perp}\right) \cdot \overline{P\left(\boldsymbol{k}_{\perp}^{\prime}\right)}\right\rangle_{B}$ in a log-log diagram with an approximation for the $\alpha=5 / 3$ case. Right: plot of the energy spectrum slopes $\alpha$ against the polarization spectrum slopes $\alpha *$ in $\left\langle P\left(\boldsymbol{k}_{\perp}\right) \cdot \overline{P\left(\boldsymbol{k}_{\perp}^{\prime}\right)}\right\rangle_{B}$.

angle $\theta$ is between the $x$-axis and vector $\boldsymbol{q}_{\perp}$, while the angle $\varphi$ rotates around the $x$-axis. Without loss of generality, we choose $\boldsymbol{k}_{\perp}=k_{\perp} e_{x}$ to ensure that the angle between $\boldsymbol{q}_{\perp}$ and $\boldsymbol{k}_{\perp}$ coincides with $\theta$. Thus the transformation is

$q_{x}=q \cos \theta$

$q_{y}=q \sin \theta \sin \varphi$

$q_{z}=q \sin \theta \cos \varphi$

which implies for the required quantities:

$$
\begin{aligned}
q_{\perp}^{2} & =q_{x}^{2}+q_{y}^{2}=q^{2} \sin ^{2} \theta \sin ^{2} \varphi+\cos ^{2} \theta=q^{2}\left(1-\sin ^{2} \theta \cos ^{2} \varphi\right) \\
a_{\perp}^{2} & =\left(\boldsymbol{q}_{\perp}+\boldsymbol{k}_{\perp}\right)^{2}=q_{\perp}^{2}+k_{\perp}^{2}+2 \boldsymbol{q}_{\perp} \cdot \boldsymbol{k}_{\perp}=q_{\perp}^{2}+k_{\perp}^{2}+2 q k_{\perp} \cos \theta \\
a^{2} & =\left(\boldsymbol{q}_{\perp}+\boldsymbol{k}_{\perp}\right)^{2}+q_{z}^{2}=q_{\perp}^{2}+k_{\perp}^{2}+q_{z}^{2}+2 \boldsymbol{q}_{\perp} \cdot \boldsymbol{k}_{\perp} \\
& =q^{2}+k_{\perp}^{2}+2 q k_{\perp} \cos \theta .
\end{aligned}
$$

The integral (52) is then transformed as follows:

$$
\begin{aligned}
K & =\int \mathrm{d} q^{3} \cdot \frac{\epsilon_{B}(q) \epsilon_{B}(a)}{q^{2} a^{2}}\left[\left(2-\frac{q_{\perp}^{2}}{q^{2}}\right)\left(2-\frac{a_{\perp}^{2}}{a^{2}}\right)\right] \\
& =\int \mathrm{d} q \int_{-1}^{1} \mathrm{~d} \cos \theta \frac{\epsilon_{B}(q) \epsilon_{B}(a)}{q^{2} a^{2}} \int_{0}^{2 \pi} \mathrm{d} \varphi\left[1+\sin ^{2} \theta \cos ^{2} \varphi\right]\left[2-\frac{q^{2}\left(1-\sin ^{2} \theta \cos ^{2} \varphi\right)+k_{\perp}^{2}+2 q k_{\perp} \cos \theta}{q^{2}+k_{\perp}^{2}+2 q k_{\perp} \cos \theta}\right] \\
& =\int \mathrm{d} q \int_{-1}^{1} \mathrm{~d} \cos \theta \frac{\epsilon_{B}(q) \epsilon_{B}(a)}{q^{2} a^{2}}\left[2+\sin ^{2} \theta+\left(1+\frac{3}{4} \sin ^{2} \theta\right)\left(\frac{q^{2} \sin ^{2} \theta}{q^{2}+k_{\perp}^{2}+2 q k_{\perp} \cos \theta}\right)\right] \pi
\end{aligned}
$$

We integrate this numerically for values of $k_{\perp}$ between $k=10^{-2}$ and $10^{3}$. We vary the spectral index $\alpha$ between $\alpha=\frac{1}{2}$ and the Kolmogorov-type spectrum $\alpha=5 / 3$. The results can be seen in Fig. 2 (left). The slope of the declining section is not equal to $\alpha$ but depends on it. It is referred to as the polarization spectrum slope $\alpha^{*}$. If one plots the energy spectrum slope $\alpha$ against the polarization spectrum slope $\alpha^{*}$, one can see that there are two different regimes, for roughly $\alpha>1$ and $\alpha<1$ (see Fig. 2 (right)).

It is now necessary to understand the approximate behavior of our findings in Fig. 2 (left), as well as the significance of the different regimes seen in Fig. 2 (right). As long as $\alpha$ is large enough, the term $\epsilon(q)$ only contributes to the integral within a sphere with radius $q_{0}$ in $q$-space, because there it is mainly constant: $\frac{\epsilon(q)}{q^{2}} \approx$ const. Beyond this sphere, it is strongly suppressed by the $q^{-\alpha}$-dependence. In this case, the slope is determined by the second term $\epsilon\left(\left|\boldsymbol{q}+\boldsymbol{k}_{\perp}^{\prime}\right|\right)$ in (59), which also contributes only within a sphere determined by $q$ around the point $k_{\perp}^{\prime}$. Inside this sphere, $\epsilon$ is roughly $\epsilon\left(k_{\perp}^{\prime}\right)$ because $q$ is smaller than $k_{\perp}^{\prime}$ since we are looking at the case $k_{\perp}^{\prime}>q_{0}$ (otherwise we get a constant behavior of the total integral as can be seen in the plots), so we get approximately $I \propto \epsilon(0) \epsilon\left(k_{\perp}^{\prime}\right)$ which leads to $\log [I] \approx-(\alpha+2) \log \left[k_{\perp}^{\prime}\right]+$ const. This is confirmed by the approximations plotted in Figs. 2 (left) and (right). In Fig. 2 (left) we plotted a rough estimate for the integral, where we had just integrated $I \propto \epsilon(0) \epsilon\left(k_{\perp}^{\prime}\right)$ inside the $q_{0^{-}}$ sphere. In order to match the original integral better, it had to be shifted by a factor of 1.6 , which is perfectly reasonable considering that the simple sphere is just an approximation for a more complex structure. In Fig. 2 (right) we see that $-\alpha-2$ is indeed a good approximation for $\alpha^{*}$ in the high- $\alpha$ regime. The regime where $\alpha<1$ is not really of physical interest because all energy spectra with $\alpha<1$ would lead to the unphysical situation of infinite energies on the smallest scales as the spectrum complies with $k^{-\alpha}$. Therefore we are not interested in the exact behavior of the integral values below $\alpha=1$. 


\section{Other correlation functions}

We now provide the results for the other correlation functions of our observables without repeating the details of the calculations. All functions of first or third order in $\boldsymbol{B}$ are omitted because they are obviously zero thanks to the uneven number of fields appearing in them. These are $\left\langle\phi\left(\boldsymbol{k}_{\perp}\right)\right\rangle_{\boldsymbol{B}},\left\langle\phi\left(\boldsymbol{k}_{\perp}\right) \phi\left(\boldsymbol{k}_{\perp}^{\prime}\right) \phi\left(\boldsymbol{k}_{\perp}^{\prime \prime}\right)\right\rangle_{\boldsymbol{B}},\left\langle I\left(\boldsymbol{k}_{\perp}\right) \phi\left(\boldsymbol{k}_{\perp}^{\prime}\right)\right\rangle_{\boldsymbol{B}}$ and $\left\langle P\left(\boldsymbol{k}_{\perp}\right) \phi\left(\boldsymbol{k}_{\perp}^{\prime}\right)\right\rangle_{\boldsymbol{B}}$. As stated before, the calculational steps to gain these expressions are fairly similar to the case of $\left\langle P\left(\boldsymbol{k}_{\perp}\right) \cdot P^{*}\left(\boldsymbol{k}_{\perp}^{\prime}\right)\right\rangle_{B}$. As a matter of fact, most of them are even easier to obtain. More complex numerical integrations are only needed for $\left\langle I\left(\boldsymbol{k}_{\perp}\right) I\left(\boldsymbol{k}_{\perp}^{\prime}\right)\right\rangle_{\boldsymbol{B}}$ and $\left\langle I\left(\boldsymbol{k}_{\perp}\right) P\left(\boldsymbol{k}_{\perp}^{\prime}\right)\right\rangle_{\boldsymbol{B}}$. In the following, the vectors $\boldsymbol{r}$ or $\boldsymbol{r}^{\prime}$ always denote a combination such as $\boldsymbol{x}^{\prime}-\boldsymbol{x}$, to be defined for each correlation function in Appendix A. By $\mathcal{B}(a, b)$ we denote the Beta-function. Furthermore $\boldsymbol{u}, \boldsymbol{v}$, and $\boldsymbol{w}$ are defined as $\boldsymbol{w}=\left(\boldsymbol{k}_{\perp}^{\prime \prime \prime}, 0\right), \boldsymbol{u}=\left(\boldsymbol{k}_{\perp}^{\prime \prime}, 0\right), \boldsymbol{v}=\left(\boldsymbol{k}_{\perp}^{\prime}, 0\right)$ and $\boldsymbol{a}=\left(-\boldsymbol{q}_{\perp}-\boldsymbol{k}_{\perp}^{\prime},-q_{z}\right)$.

The results are

$$
\begin{aligned}
& \left\langle I\left(\boldsymbol{k}_{\perp}\right)\right\rangle_{\boldsymbol{B}} \\
& =2(2 \pi)^{2} \delta^{2}\left(\boldsymbol{k}_{\perp}\right) L_{z} M_{\mathrm{N}}(0) \\
& =128 \pi^{4} \delta^{2}\left(\boldsymbol{k}_{\perp}\right) L_{z} \mathcal{B}\left(\frac{\beta}{2}+\frac{1}{2}, \frac{\alpha}{2}-\frac{1}{2}\right) \\
& \left\langle P\left(\boldsymbol{k}_{\perp}\right)\right\rangle_{\boldsymbol{B}} \\
& =0 \\
& \left\langle\phi\left(\boldsymbol{k}_{\perp}\right) \phi\left(\boldsymbol{k}_{\perp}^{\prime}\right)\right\rangle_{\boldsymbol{B}} \\
& =(2 \pi)^{2} \delta^{2}\left(\boldsymbol{k}_{\perp}+\boldsymbol{k}_{\perp}^{\prime}\right) L_{z} \hat{M}_{33}\left(\boldsymbol{k}_{\perp}^{\prime}, 0\right) \\
& =32 \pi^{5} \delta^{2}\left(\boldsymbol{k}_{\perp}+\boldsymbol{k}_{\perp}^{\prime}\right) L_{z} \epsilon(v) / v^{2} \\
& \left\langle I\left(\boldsymbol{k}_{\perp}\right) I\left(\boldsymbol{k}_{\perp}^{\prime}\right)\right\rangle_{\boldsymbol{B}} \\
& =(2 \pi)^{2} \delta^{2}\left(\boldsymbol{k}_{\perp}+\boldsymbol{k}_{\perp}^{\prime}\right) L_{z} \int \frac{\mathrm{d} q^{3}}{(2 \pi)^{3}}\left(2 \left(\hat{M}_{11}(\boldsymbol{q}) \hat{M}_{11}(\boldsymbol{a})\right.\right. \\
& \left.\left.+\hat{M}_{22}(\boldsymbol{q}) \hat{M}_{22}(\boldsymbol{a})\right)+4 \hat{M}_{21, \text { isym }}(\boldsymbol{q}) \hat{M}_{21, \text { isym }}(\boldsymbol{a})\right) \\
& =8 \pi^{2} \delta^{2}\left(\boldsymbol{k}_{\perp}+\boldsymbol{k}_{\perp}^{\prime}\right) L_{z} \int \mathrm{d} q^{3} \frac{\epsilon(q) \epsilon(a)}{q^{2} a^{2}}\left[\left(1-\frac{q_{1}^{2}}{q^{2}}\right)\left(1-\frac{a_{1}^{2}}{a^{2}}\right)\right. \\
& \left.+\left(1-\frac{q_{2}^{2}}{q^{2}}\right)\left(1-\frac{a_{2}^{2}}{a^{2}}\right)+2\left(\frac{q_{2} q_{1}}{q^{2}}\right)\left(\frac{a_{2} a_{1}}{a^{2}}\right)\right] \\
& \left\langle P\left(\boldsymbol{k}_{\perp}\right) P^{*}\left(\boldsymbol{k}_{\perp}^{\prime}\right)\right\rangle_{\boldsymbol{B}} \\
& =\frac{8}{2 \pi} \delta^{2}\left(\boldsymbol{k}_{\perp}-\boldsymbol{k}_{\perp}^{\prime}\right) L_{z} \int \mathrm{d} q^{3} \\
& \left(M_{11}(\boldsymbol{q}) M_{11}(\boldsymbol{a})+M_{22}(\boldsymbol{q}) M_{22}(\boldsymbol{a})\right. \\
& \left.+M_{22}(\boldsymbol{q}) M_{11}(\boldsymbol{a})+M_{22}(\boldsymbol{a}) M_{11}(\boldsymbol{q})\right) \\
& =8(2 \pi)^{5} \delta^{2}\left(\boldsymbol{k}_{\perp}-\boldsymbol{k}_{\perp}^{\prime}\right) L_{z} \int \mathrm{d} q^{3} \cdot \frac{\epsilon_{B}(q) \epsilon_{B}(a)}{q^{2} a^{2}} \\
& {\left[\left(2-\frac{q_{\perp}^{2}}{q^{2}}\right)\left(2-\frac{a_{\perp}^{2}}{a^{2}}\right)\right]} \\
& \left\langle\phi\left(\boldsymbol{k}_{\perp}\right) \phi\left(\boldsymbol{k}_{\perp}^{\prime}\right) \phi\left(\boldsymbol{k}_{\perp}^{\prime \prime}\right) \phi\left(\boldsymbol{k}_{\perp}^{\prime \prime \prime}\right)\right\rangle_{\boldsymbol{B}} \\
& =(2 \pi)^{4}\left[\delta^{2}\left(\boldsymbol{k}_{\perp}^{\prime \prime}+\boldsymbol{k}_{\perp}^{\prime \prime \prime}\right) \delta^{2}\left(\boldsymbol{k}_{\perp}+\boldsymbol{k}_{\perp}^{\prime}\right) \hat{M}_{\mathrm{N}}(w) \hat{M}_{\mathrm{N}}(v)\right. \\
& +\delta^{2}\left(\boldsymbol{k}_{\perp}^{\prime \prime \prime}+\boldsymbol{k}_{\perp}^{\prime}\right) \delta^{2}\left(\boldsymbol{k}_{\perp}^{\prime \prime}+\boldsymbol{k}_{\perp}\right) \hat{M}_{\mathrm{N}}(w) \hat{M}_{\mathrm{N}}(u) \\
& \left.+\delta^{2}\left(\boldsymbol{k}_{\perp}^{\prime \prime}+\boldsymbol{k}_{\perp}^{\prime}\right) \delta^{2}\left(\boldsymbol{k}_{\perp}^{\prime \prime \prime}+\boldsymbol{k}_{\perp}\right) \hat{M}_{\mathrm{N}}(w) \hat{M}_{\mathrm{N}}(u)\right] \\
& \left\langle I\left(\boldsymbol{k}_{\perp}\right) P\left(\boldsymbol{k}_{\perp}^{\prime}\right)\right\rangle_{\boldsymbol{B}} \\
& =(2 \pi)^{2} \delta^{2}\left(\boldsymbol{k}_{\perp}+\boldsymbol{k}_{\perp}^{\prime}\right) L_{z} \int \frac{\mathrm{d} q^{3}}{(2 \pi)^{3}}\left[\frac{M_{\mathrm{N}}(q) M_{\mathrm{N}}(a)}{q^{2} a^{2}}\right. \\
& \left.\left[2\left(q_{2}^{2}+q_{3}^{2}\right)\left(a_{2}^{2}+a_{3}^{2}\right)+2\left(q_{1}^{2}+q_{3}^{2}\right)\left(a_{1}^{2}+a_{3}^{2}\right)\right]\right] \\
& \left\langle I\left(\boldsymbol{k}_{\perp}\right) \phi\left(\boldsymbol{k}_{\perp}^{\prime}\right) \phi\left(\boldsymbol{k}_{\perp}^{\prime \prime}\right)\right\rangle_{\boldsymbol{B}} \\
& =L^{2}(2 \pi)^{4} \delta^{2}\left(\boldsymbol{k}_{\perp}^{\prime}+\boldsymbol{k}_{\perp}^{\prime \prime}\right) \delta^{2}\left(\boldsymbol{k}_{\perp}\right) \hat{M}_{\mathrm{N}}(u) 2 M_{\mathrm{N}}(0) \\
& -2 L_{z}(2 \pi)^{2} \delta^{2}\left(\boldsymbol{k}_{\perp}+\boldsymbol{k}_{\perp}^{\prime}+\boldsymbol{k}_{\perp}^{\prime \prime}\right) \hat{H}(u) \hat{H}(v) / u v \\
& \left(u_{1} v_{1}+u_{2} v_{2}\right) \\
& \left\langle P\left(\boldsymbol{k}_{\perp}\right) \phi\left(\boldsymbol{k}_{\perp}^{\prime}\right) \phi\left(\boldsymbol{k}_{\perp}^{\prime \prime}\right)\right\rangle_{\boldsymbol{B}} \\
& =2 L_{z}(2 \pi)^{2} \delta^{2}\left(\boldsymbol{k}_{\perp}+\boldsymbol{k}_{\perp}^{\prime}+\boldsymbol{k}_{\perp}^{\prime \prime}\right) \\
& \hat{H}(u) \hat{H}(v) / u v\left(\left(u_{1} v_{1}-u_{2} v_{2}\right)+\mathrm{i}\left(u_{1} v_{2}+u_{2} v_{1}\right)\right) \text {. }
\end{aligned}
$$

Many of the results, as expected, provide no surprises. The mean total intensity (60) is given, in principle, by the energy density of the magnetic field, whereas the mean polarized intensity (61) should be zero because of the isotropy of the problem. The correlation function (62) has already been evaluated by Enßlin \& Vogt (2003) and also by Cho \& Ryu (2009); it depends on the $k_{z}=0$ plane of $\hat{M}_{z z}$. The fourth-order quantities (63)-(65) are more complex, but nevertheless only correlated combinations of the second-order quantities. They can be used to monitor the validity of the assumption of Gaussianity and isotropy. Non-Gaussianity or anisotropy in magnetic field statistics would lead to a deviation from this form, which can be detected by comparison to $\left\langle\phi\left(\boldsymbol{k}_{\perp}\right) \phi\left(\boldsymbol{k}_{\perp}^{\prime}\right)\right\rangle_{\boldsymbol{B}}$.

By far the most interesting results are of course (67) and (68) as they contain a direct dependence on the helical power spectrum $\hat{H}(k)$. Both are plotted in Fig. 3 at the same time, but only for the case where $\boldsymbol{k}_{\perp}=0$. We also restrict ourselves to $\boldsymbol{k}=k \boldsymbol{e}_{y}$ for $(68)$ 


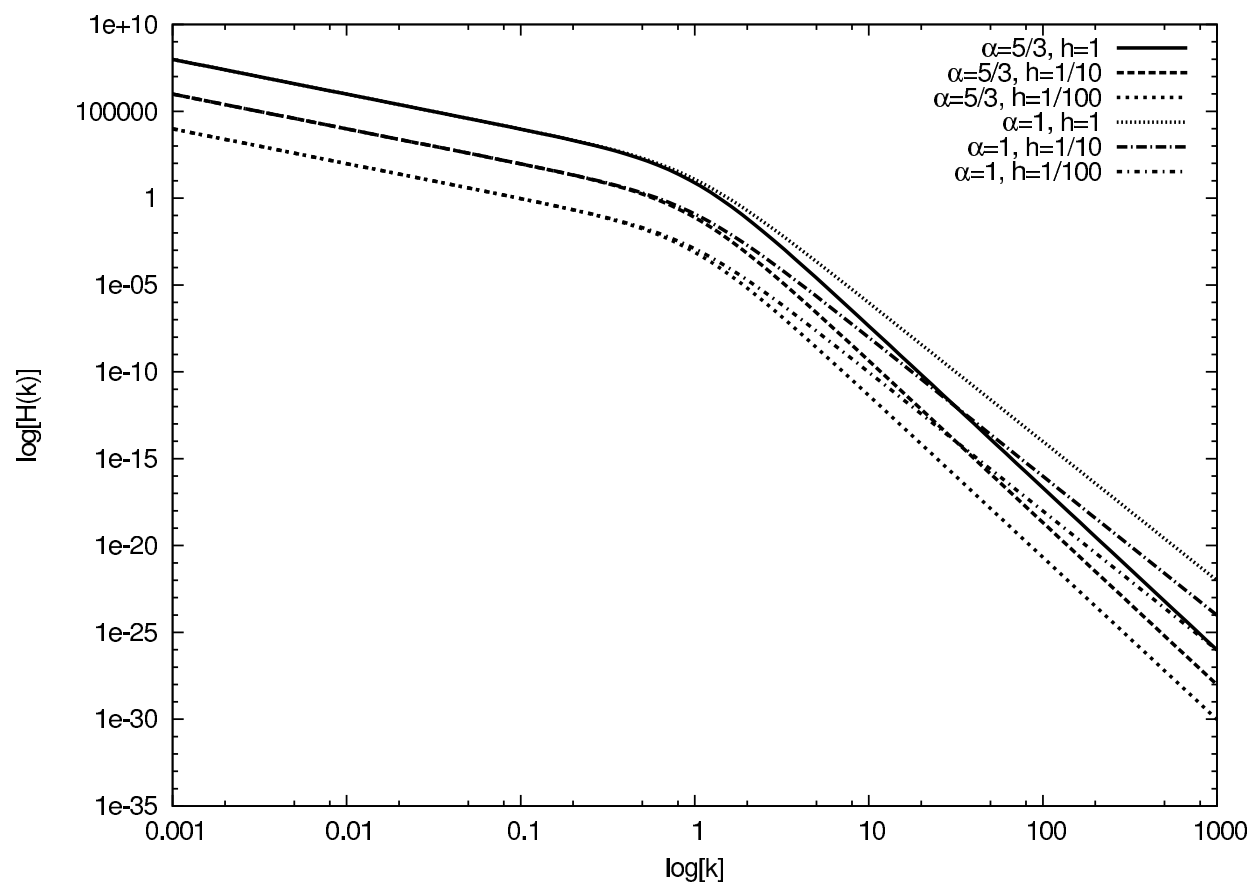

Fig. 3. Helical spectra of $\left\langle I\left(\boldsymbol{k}_{\perp}\right) \phi\left(\boldsymbol{k}_{\perp}^{\prime}\right) \phi\left(\boldsymbol{k}_{\perp}^{\prime \prime}\right)\right\rangle_{\boldsymbol{B}}$ and $\left\langle P\left(\boldsymbol{k}_{\perp}\right) \phi\left(\boldsymbol{k}_{\perp}^{\prime}\right) \phi\left(\boldsymbol{k}_{\perp}^{\prime \prime}\right)\right\rangle_{\boldsymbol{B}}$ for $\alpha=1$ and $\alpha=5 / 3, \boldsymbol{k}_{\perp}=0$ and different values of $h(k)$ and under further assumptions for which both functions take on the same analytical form.

without loss of generality. This reduces both correlation functions to $2(2 \pi)^{2} \hat{H}^{2}(k)$. Actually plotted is only $\hat{H}^{2}(k)$. To represent the helical spectrum $\hat{H}^{2}(k)$ graphically, we factor out the energy spectrum $\epsilon_{B}(k)$ thus leaving us a function $h(k)$, that parameterizes the plots (see Sect. 2.2).

The exact results (67) and (68) can be understood from the physical point of view. In both correlation functions, due to the polarization properties of synchrotron emission, we have a part connected to the magnetic field component that lies in the surface perpendicular to the line of sight $I\left(\boldsymbol{k}_{\perp}\right)$ and $P\left(\boldsymbol{k}_{\perp}\right)$. There is also a part that depends on the line-of-sight component $\phi\left(\boldsymbol{k}_{\perp}\right) \phi\left(\boldsymbol{k}_{\perp}^{\prime}\right)$ owing to Faraday rotation. If we consider the corresponding delta functions, we see that in cases, where we have $\delta^{2}\left(\boldsymbol{k}_{\perp}^{\prime}+\boldsymbol{k}_{\perp}^{\prime \prime}\right) \delta^{2}\left(\boldsymbol{k}_{\perp}\right)$ the two parts are somewhat uncorrelated as the related term is more or less the product of $\left\langle I\left(\boldsymbol{k}_{\perp}\right)\right\rangle_{\boldsymbol{B}}$ and $\left\langle\phi\left(\boldsymbol{k}_{\perp}\right) \phi\left(\boldsymbol{k}_{\perp}^{\prime}\right)\right\rangle_{\boldsymbol{B}}$. This is reflected in the absence of any helicity dependence. In contrast, if we examine the parts with $\delta^{2}\left(\boldsymbol{k}_{\perp}+\boldsymbol{k}_{\perp}^{\prime}+\boldsymbol{k}_{\perp}^{\prime \prime}\right)$, there is a type of mixing between the different observables caused by the mutual dependence of the three vectors $\boldsymbol{k}_{\perp}, \boldsymbol{k}_{\perp}^{\prime}, \boldsymbol{k}_{\perp}^{\prime \prime}$ through the delta function. Accordingly, there is a dependence on helicity. More about the physical interpretation of these results can be found in the following section.

Please note that information on the overall sign of the helicity cannot be obtained using this method becaus $\hat{H}(k)$ only appears quadratically.

\section{The LITMUS test}

For the correlation function (68), a strikingly intuitive picture can be found to explain the result. Let us take a look at Fig. 4 where we imagine the line of sight to be directly aligned with the axis of a magnetic helix. In a combined polarization and Faraday depth map, we should see a central region with nonzero faraday depth $\phi$ and around it a radial polarization pattern. These are correlated structures, which make (68) nonzero for helical magnetic fields. They would vanish with the field becoming nonhelical. However, we clearly have two possibilities for the direction of the magnetic field going around and therefore could get positive or negative $\phi$, respectively. Thus, these correlated structures can only be seen in $\left\langle P\left(\boldsymbol{k}_{\perp}\right) \phi\left(\boldsymbol{k}_{\perp}^{\prime}\right) \phi\left(\boldsymbol{k}_{\perp}^{\prime \prime}\right)\right\rangle_{\boldsymbol{B}}$ and not in $\left\langle P\left(\boldsymbol{k}_{\perp}\right) \phi\left(\boldsymbol{k}_{\perp}^{\prime}\right)\right\rangle_{\boldsymbol{B}}$, where the single dependence on $\phi$ would induce the positive and negative parts to cancel out over averaging. This is confirmed, because $\left\langle P\left(\boldsymbol{k}_{\perp}\right) \phi\left(\boldsymbol{k}_{\perp}^{\prime}\right)\right\rangle_{\boldsymbol{B}}$ becomes zero due to the odd number of functional derivatives. helicity.

Guided by this picture, the LITMUS test was developed, a short and simple test that could be easily used to probe real data for

It was not constructed to produce quantitative measurements of the helicity spectra, but to provide a fast and qualitative test for the presence of helicity.

\subsection{The basic idea}

We take a closer look at one of the aforementioned patterns of polarization and Faraday rotation (see Fig. 4). In the ideal, helical case, the gradient $\mathbf{G}=\nabla \phi$ of $\phi$ points either to the center or out of the center of the region with Faraday rotation and should therefore be perfectly aligned with the polarization. The polarized intensity $P$ is a complex number representing a spin 2 field. To compare $P$ with $\mathbf{G}$, we simply transform $\mathbf{G}$ from a 2 D vector into a complex number $\mathcal{G}$ in the same representation of "directionless vectors":

$\mathcal{G}=|\mathbf{G}|^{2} \exp [2 \mathrm{i} \alpha] \quad$ with $\alpha=\arctan \frac{G_{y}}{G_{x}}$. 

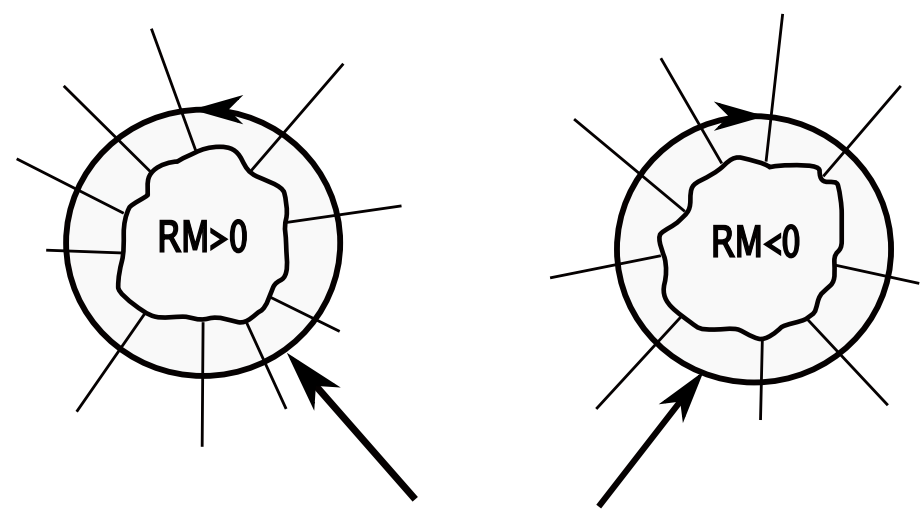

\section{Helical magnetic fieldlines \\ in two different directios}

Fig. 4. Schematic picture of correlated structures in combined polarization and RM maps that can give rise to a non-zero correlation function $\left\langle P\left(\boldsymbol{k}_{\perp}\right) \phi\left(\boldsymbol{k}_{\perp}^{\prime}\right) \phi\left(\boldsymbol{k}_{\perp}^{\prime \prime}\right)\right\rangle_{\boldsymbol{B}}$

By doing so, we lose the information about the direction that the gradient $\mathbf{G}$ is pointing. The quadratic dependence of $\mathcal{G}$ on $|\mathbf{G}|$ accounts for a normalization that will become clear in the following.

We now want to construct a test using $\mathcal{G}$ and $P$ that is sensitive to the presence of magnetic helicity but can be performed easily on a real dataset. Our previous considerations suggest to simply using the need for $\mathcal{G}$ and $P$ to be parallel for helical fields. The test then only consists of multiplying $\mathcal{G}$ with $P^{*}$ for every pixel of a given map of $\phi^{2}$ and $P$. This complex scalar product produces different results for different orientations of $\mathcal{G}$ and $P$ in the complex plane. If the gradient and the polarization are parallel $(\chi$ and $\alpha$ differ by a multiple of $\pi$ ), the result is real and positive. If they are perpendicular $(\chi$ and $\alpha$ differ by an odd multiple of $\pi / 2)$ the result is real and negative. For any orientations in between, the result will take on complex values.

We now can outline the LITMUS test. In the presence of helical fields, the average of the scalar product $\mathcal{G}^{*} P$ over all pixels of a $\mathcal{G}^{*} P$-map should have a real value that is significantly higher than zero. Whereas in the case of nonhelical fields, the alignment of $\mathcal{G}$ and $P$ should be changing randomly from pixel to pixel so that we would expect the average over $\mathcal{G}^{*} P$ to be zero. In short mathematical notation this is stated as

$\left\langle\mathcal{G}^{*} P\right\rangle_{\text {helicity }}>0$ and real,

$\left\langle\mathcal{G}^{*} P\right\rangle_{\text {no helicity }}=0$,

where the ensemble average over helical or nonhelical fields is in practice replaced by an average over all pixels of a $\mathcal{G}^{*} P$-map.

It can be shown that our intuition was right and that this test actually depends directly on $\left\langle P(\boldsymbol{k}) \phi\left(\boldsymbol{k}^{\prime}\right) \phi\left(\boldsymbol{k}^{\prime \prime}\right)\right\rangle$, which was our starting point. We begin by writing out the full condition for the LITMUS test:

$\left\langle\left(\mathcal{G}^{*}(\boldsymbol{x}) P(\boldsymbol{x})\right)\right\rangle=\left\langle\left[\left(\frac{\partial \phi(\boldsymbol{x})}{\partial x}\right)^{2}+\left(\frac{\partial \phi(\boldsymbol{x})}{\partial y}\right)^{2}\right] \exp \left[-2 \mathrm{i} \arctan G_{y} / G_{x}\right] P(\boldsymbol{x})\right\rangle$.

Using trigonometrical theorems, it is easy to show that

$\left(G_{x}^{2}+G_{y}^{2}\right) \exp \left[-2 \mathrm{i} \arctan G_{y} / G_{x}\right]=\left(G_{x}-\mathrm{i} G_{y}\right)^{2}$

We now apply a Fourier transformation to our observables $P(x)$ and $\phi(x)$ and rewrite the whole expression:

$$
\begin{aligned}
\left\langle\mathcal{G}^{*}(\boldsymbol{x}) P(\boldsymbol{x})\right\rangle & =\left\langle\left[\frac{\partial \phi(\boldsymbol{x})}{\partial x_{1}}-\mathrm{i} \frac{\partial \phi(\boldsymbol{x})}{\partial x_{2}}\right]^{2} P(\boldsymbol{x})\right\rangle \\
& =\left\langle\left(\left[\frac{\partial}{\partial x_{1}}-\mathrm{i} \frac{\partial}{\partial x_{2}}\right] \phi(\boldsymbol{x})\right)\left(\left[\frac{\partial}{\partial x_{1}}-\mathrm{i} \frac{\partial}{\partial x_{2}}\right] \phi(\boldsymbol{x})\right) P(\boldsymbol{x})\right\rangle \\
& =\left\langle\int \frac{\mathrm{d} k^{2}}{(2 \pi)^{2}} \int \frac{\mathrm{d} k^{2^{\prime}}}{(2 \pi)^{2}} \int \frac{\mathrm{d} k^{2^{\prime \prime}}}{(2 \pi)^{2}}\left[k_{1}^{\prime}-\mathrm{i} k_{2}^{\prime}\right]\left[k_{1}^{\prime \prime}-\mathrm{i} k_{2}^{\prime \prime}\right] \exp \left[\mathrm{i} \boldsymbol{x}\left(\boldsymbol{k}+\boldsymbol{k}^{\prime}+\boldsymbol{k}^{\prime \prime}\right)\right] P(\boldsymbol{k}) \phi\left(\boldsymbol{k}^{\prime}\right) \phi\left(\boldsymbol{k}^{\prime \prime}\right)\right\rangle \\
& =\int \frac{\mathrm{d} k^{2}}{(2 \pi)^{2}} \cdots \int \frac{\mathrm{d} k^{2^{\prime \prime}}}{(2 \pi)^{2}}\left[k_{1}^{\prime}-\mathrm{i} k_{2}^{\prime}\right]\left[k_{1}^{\prime \prime}-\mathrm{i} k_{2}^{\prime \prime}\right] \exp \left[\mathrm{i} \boldsymbol{x}\left(\boldsymbol{k}+\boldsymbol{k}^{\prime}+\boldsymbol{k}^{\prime \prime}\right)\right] \\
& \left\langle P(\boldsymbol{k}) \phi\left(\boldsymbol{k}^{\prime}\right) \phi\left(\boldsymbol{k}^{\prime \prime}\right)\right\rangle .
\end{aligned}
$$

We assume again that the average over all pixels of a $\mathcal{G}^{*} P$-map is equivalent to an ensemble average over the magnetic field statistics. 
Now we insert our result (68) for $\left\langle P(\boldsymbol{k}) \phi\left(\boldsymbol{k}^{\prime}\right) \phi\left(\boldsymbol{k}^{\prime \prime}\right)\right\rangle$ and see the dependence of $\left\langle\mathcal{G}(\boldsymbol{x}) P^{*}(\boldsymbol{x})\right\rangle$ on the helical spectra $\hat{H}\left(k^{\prime}\right) \hat{H}\left(k^{\prime \prime}\right)$ :

$$
\begin{aligned}
\left\langle\mathcal{G}(\boldsymbol{x}) P^{*}(\boldsymbol{x})\right\rangle= & 2 L_{z}(2 \pi)^{2} \int \frac{\mathrm{d} k^{2}}{(2 \pi)^{2}} \cdots \int \frac{\mathrm{d} k^{2^{\prime \prime}}}{(2 \pi)^{2}} \delta^{2}\left(\boldsymbol{k}+\boldsymbol{k}^{\prime}+\boldsymbol{k}^{\prime \prime}\right)\left[k_{1}^{\prime}-\mathrm{i} k_{2}^{\prime}\right]\left[k_{1}^{\prime \prime}-\mathrm{i} k_{2}^{\prime \prime}\right] \\
& \exp \left[\mathrm{i} \boldsymbol{x}\left(\boldsymbol{k}+\boldsymbol{k}^{\prime}+\boldsymbol{k}^{\prime \prime}\right)\right]\left[\left(k_{1}^{\prime} k_{1}^{\prime \prime}-k_{2}^{\prime} k_{2}^{\prime \prime}\right)+\mathrm{i}\left(k_{1}^{\prime} k_{2}^{\prime \prime}+k_{2}^{\prime} k_{1}^{\prime \prime}\right)\right] \frac{\hat{H}\left(k^{\prime}\right) \hat{H}\left(k^{\prime \prime}\right)}{k^{\prime} k^{\prime \prime}} \\
= & 2 L_{z}(2 \pi)^{2} \int \frac{\mathrm{d} k^{2^{\prime}}}{(2 \pi)^{2}} \int \frac{\mathrm{d} k^{2^{\prime \prime}}}{(2 \pi)^{2}}\left[k_{1}^{\prime}-\mathrm{i} k_{2}^{\prime}\right]\left[k_{1}^{\prime \prime}-\mathrm{i} k_{2}^{\prime \prime}\right]\left[\left(k_{1}^{\prime} k_{1}^{\prime \prime}-k_{2}^{\prime} k_{2}^{\prime \prime}\right)+\mathrm{i}\left(k_{1}^{\prime} k_{2}^{\prime \prime}+k_{2}^{\prime} k_{1}^{\prime \prime}\right)\right] \\
& \frac{\hat{H}\left(k^{\prime}\right) \hat{H}\left(k^{\prime \prime}\right)}{k^{\prime} k^{\prime \prime}} \\
= & 2 L_{z}(2 \pi)^{2} \int \frac{\mathrm{d} k^{2^{\prime}}}{(2 \pi)^{2}} \int \frac{\mathrm{d} k^{2^{\prime \prime}}}{(2 \pi)^{2}}\left[k_{1}^{2^{\prime}}+k_{2}^{2^{\prime}}\right]\left[k_{1}^{2^{\prime \prime}}+k_{2}^{2^{\prime \prime}}\right] \frac{\hat{H}\left(k^{\prime}\right) \hat{H}\left(k^{\prime \prime}\right)}{k^{\prime} k^{\prime \prime}} \\
= & 2 L_{z}(2 \pi)^{2} \int \frac{\mathrm{d} k^{\prime}}{(2 \pi)^{2}} \int \frac{\mathrm{d} k^{2^{\prime \prime}}}{(2 \pi)^{2}} k^{\prime} k^{\prime \prime} \hat{H}\left(k^{\prime}\right) \hat{H}\left(k^{\prime \prime}\right) \\
= & 2 L_{z}\left[\int_{0}^{\infty} \mathrm{d} k k^{2} \hat{H}(k)\right]^{2}=2 L_{z} \pi^{4}\left[\int_{0}^{\infty} \mathrm{d} k \frac{\epsilon_{\mathrm{H}}(k)}{k}\right]^{2} .
\end{aligned}
$$

In (75) we assumed w. 1. of g. that $\boldsymbol{x}=0$, and in the last line we used (17) to substitute $\epsilon_{\mathrm{H}}$. We see that $\left\langle\mathcal{G}(\boldsymbol{x}) P^{*}(\boldsymbol{x})\right\rangle$ is a direct and clear estimator that measures the square of the $\mathrm{k}$-space-integrated helicity spectrum, giving more weight to the large scales. Therefore, we conclude that, except perhaps from pathological or fine-tuned situations in which the k-weighted helicities on different k-scales cancel each other out (since $\hat{H}(k)$ might change sign), we can expect the LITMUS test to be able to reveal the presence of magnetic helicity. This is demonstrated in numerical tests on simulated data conducted by Oppermann et al. (2010). This work also contains an application to real data of our own galaxy and a thorough analysis thereof ${ }^{2}$.

Finally, we can state that, in principle, a given dataset can be tested for helicity. If $P$ and $\phi$ are available, a test using conditions (70) and (71) can easily be implemented caused by using local quantities alone, avoiding any data transformation to Fourier space and the complication finite window functions thereby would introduce.

\section{Conclusions}

We have shown how statistical properties of turbulent cosmic magnetic fields can be imprinted on the statistics of certain radio observables. Our analysis involved the total intensity $I\left(\boldsymbol{k}_{\perp}\right)$ and the polarized intensity $P\left(\boldsymbol{k}_{\perp}\right)$ coming from radio synchrotron emission out of a volume, as well as the Farady depth $\phi\left(\boldsymbol{k}_{\perp}\right)$ of background sources seen through the same volume. The first two depend on $B_{\perp}$, the magnetic field component lying in the surface perpendicular to the line of sight. In contrast, $\phi\left(\boldsymbol{k}_{\perp}\right)$ depends on $B_{\|}$, the component parallel to the line of sight. Whenever this set of observables is available, we can examine all three components of the magnetic field.

With regard to these observables, we evaluated a complete set of cross-correlation functions up to fourth order in the magnetic field and presented simple analytical equations in Fourier space depending on the field's energy spectra. We demonstrated that two correlation functions of our set, namely $\left\langle P\left(\boldsymbol{k}_{\perp}\right) \phi\left(\boldsymbol{k}_{\perp}^{\prime}\right) \phi\left(\boldsymbol{k}_{\perp}^{\prime \prime}\right)\right\rangle_{\boldsymbol{B}}$ and $\left\langle I\left(\boldsymbol{k}_{\perp}\right) \phi\left(\boldsymbol{k}_{\perp}^{\prime}\right) \phi\left(\boldsymbol{k}_{\perp}^{\prime \prime}\right)\right\rangle_{\boldsymbol{B}}$, explicitly depend on the helical spectra of the turbulent field. The first one depends solely on the helical parts and becomes zero for non-helical fields.

This finding offers a new way for measuring the helicity of magnetic fields and thereby for testing existing mean field dynamo theories involving helicity. Measuring these correlation functions in real data will permit the study of helicity spectra up to their overall sign. If (67) or (68) provide a non-zero result with statistical significance for $\boldsymbol{k}_{\perp} \neq 0$, this is direct evidence for helicity in the magnetic field.

Furthermore, we presented the LITMUS test, a simple procedure to be applied to data which probes for helicity. The LITMUS test is easy to apply since it can be fully computed in real space. It provides the square of the $k$-weighted $k$-space integrated helicity. First results of an application of the test to real data and to numerical tests using simulated helical and non-helical fields can be found in Oppermann et al. (2010).

Our general formalism permits the construction of further tests which can probe helicity on invidual k-scales. However, before such tests can be applied to real data, suitable observational configurations and further theoretical development in order to alleviate the simplifications and assumptions made beforehand are required.

Subsequent work should therefore follow two directions: to find observations that match our assumptions best and to extend our calculations to be able to cope with more complex observational situations. Advancements should include

- more realistic non-Gaussian components of the magnetic field statistics;

- the removal of statistical homogeneity as an overall simplification;

- spatially varying electron densities instead of assuming them as constant (see Appendix B);

- the possibility for preciser values for the spectral index of the cosmic ray electron density than the choice $p=3$ (see Appendix B);

\footnotetext{
${ }^{2}$ Unfortunately, it seems that a straightforward application of this test can be hampered by a too large spatial variance in the elctron density, which we assumed to be constant in this work.
} 
- calculations without the restrictions imposed by observed space being large leading to the approximation $\int_{\text {source }}^{\text {observer }} \mathrm{d} z \approx \int_{-\infty}^{\infty} \mathrm{d} z$ (see Sect. 2.3);

- the introduction of a window function formalism;

- and finally developing an approach for dealing with intrinsic Faraday rotation which modifies the polarization at long wavelengths, a topic which has been neglected here (see Appendix B).

Before becoming more deeply involved in discussions on possible advances in the future, we should first think about which observations could be applicable to our approach in the present form. Observations required by our analysis have to come from a polarized radio-synchrotron source with background Faraday-rotation. A suitable target could probably be found in the interstellar medium (ISM) within our own galaxy, of which we havesome established knowledge about the large-scale fields. With regard to the latter point, it would be advantageous to choose a region in which the magnetic helicity flows are expected to be found in accordance with mean field theory. Furthermore, the polarization data has to be taken at high frequencies to be Faraday rotation free. For that, the upcoming Planck polarization data of our Galaxy will be ideal, since it is at a short wavelength, has high resolution and accuracy, and is also full sky. Compilations of RM measurements of background sources seen through our galaxy already exist (Haverkorn 2007; Brown et al. 2007; Taylor et al. 2009).

Promising extragalactic objects to be investigated for magnetic field statistics are the lobes of radio galaxies, whose intensity and polarization statistics can be constructed. However, no Faraday rotation could be detected yet through their lobes. For the radio jets, this is different and, there, helicity can be probed and is actually expected to be present (Enßlin 2003; Gabuzda 2005; Mahmud \& Gabuzda 2008).

Galaxy clusters are probably not suited to our approach, although they host large-scale magnetic fields. The high degree of intrinsic Faraday rotation usually found there erases polarization of the cluster radio halo emission at the synchrotron frequencies where we observe them. However, handling intrinsic Faraday-rotation analytically is a considerable challenge. We would need to include an extra exponential factor for the rotation in $P\left(\boldsymbol{x}_{\perp}\right)$ :

$P\left(\boldsymbol{x}_{\perp}\right)=\int \mathrm{d} z \cdot\left[B_{1}(\boldsymbol{x})+\mathrm{i} B_{2}(\boldsymbol{x})\right]^{2} \cdot \exp \left[2 \mathrm{i} \phi\left(\boldsymbol{x}_{\perp}\right) \lambda^{2}\right]$.

As $\phi\left(\boldsymbol{x}_{\perp}\right)$ itself contains an integration over $\mathrm{d} z$, the extra exponential factor couples all positions along the line of sight. Thus, the exponential becomes so complicated that it has to be approximated in a suitable way. This seems to spoil a purely analytical approach. Nevertheless, including intrinsic Faraday rotation is one of the next important challenges because it would enable our approach to be applied to many sources excluded until now, such as galaxy clusters.

To further summarize our basic discussion, there is more to consider. From a technical aspect, the first problem to be tackled for tests that aim to measure detailed magnetic energy and helicity spectra, is to include a realistic window function in the formalism. A window function is set by the observations but also incorporates variation in the signal due to changing relativistic electron density and magnetic field strength. It scales with the electron density $n_{\mathrm{e}}(\boldsymbol{x})$ for $\phi\left(\boldsymbol{k}_{\perp}\right)$, with the cosmic ray electron density $n_{\text {cre }}(\boldsymbol{x})$ for $I\left(\boldsymbol{k}_{\perp}\right)$ and $P\left(\boldsymbol{k}_{\perp}\right)$, and with the average magnetic field profile. The two first have to be taken from independent observations (e.g. free-free emission), while the last has to be guessed by the source and by prior knowledge. It is clear that introducing a window function will make calculation and integration more complex. In principle, however, there is no basic restriction to its implementation. Previous attempts to measure magnetic power spectra from observations of magnetic fields heavily affected by window functions have already proven successful, e.g. Enßlin \& Vogt (2003), Vogt \& Enßlin (2005) and Kuchar \& Enßlin (2009).

The next aspect to be considered is whether to use Gaussian statistics. Real fields are probably non-Gaussian. Any attempt to model the real situation exactly has to at least include non-Gaussian deviations. This brings additional complexity into our approach as they cannot be handled analytically anymore. In (34), we would not get rid of the path integral and would need to rely on approximations or perturbative approaches, as in field theory.

At the beginning, our primary goal was to find simple analytical relationships between the statistics of radio observables and magnetic fields and the second to prove it conceptually with this approach, it is in principle possible to extract information about the helical part of the magnetic field. To show this, we therefore chose to start with Gaussian statistics, the simplest configuration possible, which also would allow us to keep our calculations analytical, while higher order statistics always could be incorporated later in a perturbative expansion around the Gaussian case. We do not claim that this assumption is sufficient to reproduce exact results in accordance with the high complexity of real nature. However, we do believe that while the grade of Gaussianity in our statistics might determine the strength with which the statistics of observables depends on magnetic properties like helicity, the assumption of Gaussianity is not essential for the dependence itself to occur. Thus we believe we should be able to decide whether data contains signatures of helical magnetic fields or not and that the simplification of using Gaussian fields in our calculations is sufficient to achieve the goal of showing how helicity and other magnetic properties can be detected. This of course needs to be shown, e.g., using mock data from numerical simulations, but this is left for later studies.

The reminder of our assumptions and simplifications only represent minor problems. Deviations from the cosmic ray electrons spectral index $p=3$ could be included in the form of correction terms. To assume that the distance between observer and source is very large(and therefore the line of sight projection parallel) usually a fairly good approximation given the vast distances we encounter on cosmic scales, but it might break down if we analyze the large-scale magnetic field directly in front of us. For the LITMUS test, the work of Oppermann et al. (2010) shows that it is still applicable in such a situation; however the varying $n_{\mathrm{e}}$ seems to be a more severe problem. The assumption of statistical homogeneity is widely used in the literature and has proved appropriate for similar problems in the past.

The outcome of our study will hopefully contribute to new findings on cosmic magnetic fields. It establishes a new, structured, and definite way to measure magnetic helicity, and it offers a new option for testing cosmic dynamo theories. The LITMUS test might be able to easily probe data for helicity. However, first results by Oppermann et al. (2010) show us that further development is 
necessary for an actual helicity-sensitive implementation, given the complication in realistic observational situations, especially the varying electron density. Last but not least, we presented a complete range of correlation functions of radio observables in elegant, simple forms, which are easy to evaluate and, in principle, can be compared to real data. In fact, some of the correlation functions may be of interest in themselves, when disregarding the topic of helicity.

Acknowledgements. This research was performed in the framework of the DFG Forschergruppe 1254 "Magnetisation of Interstellar and Intergalactic Media: The Prospects of Low-Frequency Radio Observations". The idea for this work emerged from the very stimulating discussion with Rodion Stepanov during his visit to Germany, which was supported by the DFG-RFBR grant 08-02-92881. We would like to thank Cornelius Weig, Niels Oppermann, Georg Robbers, and an anonymous referee for helpful discussions and careful reading of our manuscripts.

\section{Appendix A: All correlation functions}

This appendix contains a full list of all evaluated correlation functions and their derivations. This constitutes the core of our work, but provides no further inside information on the concept, nor does it help understanding the findings. Therefore, we have collected the calculations in this Appendix. The details of the calculations are similar to the example of $\left\langle P\left(\boldsymbol{k}_{\perp}\right) \cdot P^{*}\left(\boldsymbol{k}_{\perp}^{\prime}\right)\right\rangle_{B}$, dealt within some depth in Sect. 3 and are only commented on if necessary. All correlation functions are given in Fourier space. The functions with an odd number of fields are equal to zero due to the analogous odd number of derivatives with respect to the generating functional $\boldsymbol{J}$. The calculations involving $P\left(\boldsymbol{k}_{\perp}\right)$ are given in a Faraday-free case, but set up in such a way that we could include Faraday rotational effects for further analysis later on. Refer to Sect. 6 for more information on future perspectives. In the following, the vector $\boldsymbol{r}$ or $\boldsymbol{r}^{\prime}$ always denotes a combination such as $\boldsymbol{x}^{\prime}-\boldsymbol{x}$, to be defined for each correlation function in Appendix A. In the following $\boldsymbol{u}, \boldsymbol{v}$, and $\boldsymbol{w}$ are defined as $\boldsymbol{w}=\left(\boldsymbol{k}_{\perp}^{\prime \prime \prime}, 0\right), \boldsymbol{u}=\left(\boldsymbol{k}_{\perp}^{\prime \prime}, 0\right), \boldsymbol{v}=\left(\boldsymbol{k}_{\perp}^{\prime}, 0\right)$, and $\boldsymbol{a}=\left(-\boldsymbol{q}_{\perp}-\boldsymbol{k}_{\perp}^{\prime},-q_{z}\right)$.

\section{A.1. Calculation of $\left\langle\phi\left(\boldsymbol{k}_{\perp}\right)\right\rangle_{\boldsymbol{B}}$}

$\left\langle\phi\left(\boldsymbol{k}_{\perp}\right)\right\rangle_{\boldsymbol{B}}=\left.\int \mathrm{d} x^{3} \exp \left[\mathrm{i} \boldsymbol{k}_{\perp} \boldsymbol{x}_{\perp}\right] \partial_{3}(\boldsymbol{x}) \exp \left[\frac{1}{2} \boldsymbol{J}^{\dagger} \mathbf{M} \boldsymbol{J}\right]\right|_{\boldsymbol{J}=0}=0$.

\section{A.2. Calculation of $\left\langle\phi\left(\boldsymbol{k}_{\perp}\right) \phi\left(\boldsymbol{k}_{\perp}^{\prime}\right)\right\rangle_{\boldsymbol{B}}$}

$$
\begin{aligned}
\left\langle\phi\left(\boldsymbol{k}_{\perp}\right) \phi\left(\boldsymbol{k}_{\perp}^{\prime}\right)\right\rangle_{\boldsymbol{B}}=\int \mathrm{d} x^{3} \int \mathrm{d} x^{\prime 3} \exp \left[\mathrm{i} \boldsymbol{k}_{\perp} \boldsymbol{x}_{\perp}+\mathrm{i} \boldsymbol{k}_{\perp}^{\prime} \boldsymbol{x}_{\perp}^{\prime}\right] \partial_{3}(\boldsymbol{x}) \partial_{3}\left(\boldsymbol{x}^{\prime}\right) \\
\left.\exp \left[\frac{1}{2} \boldsymbol{J}^{\dagger} \mathbf{M} \boldsymbol{J}\right]\right|_{\boldsymbol{J}=0} \\
\quad=\int \mathrm{d} x^{3} \int \mathrm{d} x^{\prime 3} \exp \left[\mathrm{i} \boldsymbol{k}_{\perp} \boldsymbol{x}_{\perp}+\mathrm{i} \boldsymbol{k}_{\perp}^{\prime} \boldsymbol{x}_{\perp}^{\prime}\right] M_{33}(\boldsymbol{r}) \\
=\int \mathrm{d} x^{3} \int \mathrm{d} r^{3} \exp \left[\mathrm{i} \boldsymbol{x}_{\perp}\left(\boldsymbol{k}_{\perp}+\boldsymbol{k}_{\perp}^{\prime}\right)\right] \exp \left[\mathrm{i} \boldsymbol{r}_{\perp} \boldsymbol{k}_{\perp}^{\prime}\right] M_{33}(\boldsymbol{r}) \\
=(2 \pi)^{2} \delta^{2}\left(\boldsymbol{k}_{\perp}+\boldsymbol{k}_{\perp}^{\prime}\right) L_{z} \int \mathrm{d} r^{3} \exp \left[\mathrm{i} \boldsymbol{r}_{\perp} \boldsymbol{k}_{\perp}^{\prime}\right] M_{33}(\boldsymbol{r}) \\
=(2 \pi)^{2} \delta^{2}\left(\boldsymbol{k}_{\perp}+\boldsymbol{k}_{\perp}^{\prime}\right) L_{z} \int \mathrm{d} r^{3} \int \frac{\mathrm{d} q^{3}}{(2 \pi)^{3}} \exp \left[\mathrm{i} \boldsymbol{r}_{\perp} \boldsymbol{k}_{\perp}^{\prime}\right] \exp [-\mathrm{i} \boldsymbol{r} \boldsymbol{q}] \hat{M}_{33}(\boldsymbol{q}) \\
=(2 \pi)^{2} \delta^{2}\left(\boldsymbol{k}_{\perp}+\boldsymbol{k}_{\perp}^{\prime}\right) L_{z} \int \mathrm{d} q^{3} \delta^{2}\left(\boldsymbol{k}_{\perp}^{\prime}-\boldsymbol{q}_{\perp}\right) \delta\left(-q_{z}\right) \hat{M}_{33}(\boldsymbol{q}) \\
=(2 \pi)^{2} \delta^{2}\left(\boldsymbol{k}_{\perp}+\boldsymbol{k}_{\perp}^{\prime}\right) L_{z} \hat{M}_{33}\left(\boldsymbol{k}_{\perp}^{\prime}, 0\right) \\
=(2 \pi)^{2} \delta^{2}\left(\boldsymbol{k}_{\perp}+\boldsymbol{k}_{\perp}^{\prime}\right) L_{z} \hat{M}_{\mathrm{N}}(v) \\
=32 \pi^{5} \delta^{2}\left(\boldsymbol{k}_{\perp}+\boldsymbol{k}_{\perp}^{\prime}\right) L_{z} \frac{\epsilon(v)}{v^{2}} .
\end{aligned}
$$

\section{A.3. Calculation of $\left\langle\phi\left(\boldsymbol{k}_{\perp}\right) \phi\left(\boldsymbol{k}_{\perp}^{\prime}\right) \phi\left(\boldsymbol{k}_{\perp}^{\prime \prime}\right)\right\rangle_{\boldsymbol{B}}$}

$\left\langle\phi\left(\boldsymbol{k}_{\perp}\right) \phi\left(\boldsymbol{k}_{\perp}^{\prime}\right) \phi\left(\boldsymbol{k}_{\perp}^{\prime \prime}\right)\right\rangle_{\boldsymbol{B}}=a_{0}^{3} n_{\mathrm{e}}^{3} \int \mathrm{d} x^{3} \int \mathrm{d} x^{\prime 3} \int \mathrm{d} x^{\prime \prime 3} \exp \left[\mathrm{i} \boldsymbol{k}_{\perp} \boldsymbol{x}_{\perp}+\mathrm{i} \boldsymbol{k}_{\perp}^{\prime} \boldsymbol{x}_{\perp}^{\prime}+\boldsymbol{k}_{\perp}^{\prime \prime} \boldsymbol{x}_{\perp}^{\prime \prime}\right]$
$\left.\left.\left.\partial_{3}(\boldsymbol{x})\right|_{\boldsymbol{J}=0} \partial_{3}\left(\boldsymbol{x}^{\prime}\right)\right|_{\boldsymbol{J}=0} \partial_{3}\left(\boldsymbol{x}^{\prime \prime}\right) \exp \left[\frac{1}{2} \boldsymbol{J}^{\dagger} \mathbf{M} \boldsymbol{J}\right]\right|_{\boldsymbol{J}=0}=0$. 


\section{A.4. Calculation of $\left\langle\phi\left(\boldsymbol{k}_{\perp}\right) \phi\left(\boldsymbol{k}_{\perp}^{\prime}\right) \phi\left(\boldsymbol{k}_{\perp}^{\prime \prime}\right) \phi\left(\boldsymbol{k}_{\perp}^{\prime \prime \prime}\right)\right\rangle_{\boldsymbol{B}}$}

$$
\begin{aligned}
&\left\langle\phi\left(\boldsymbol{k}_{\perp}\right) \phi\left(\boldsymbol{k}_{\perp}^{\prime}\right) \phi\left(\boldsymbol{k}_{\perp}^{\prime \prime}\right) \phi\left(\boldsymbol{k}_{\perp}^{\prime \prime \prime}\right)\right\rangle_{\boldsymbol{B}}=n_{\mathrm{e}}^{4} \int \mathrm{d} x^{3} \int \mathrm{d} x^{\prime 3} \int \mathrm{d} x^{\prime \prime 3} \int \mathrm{d} x^{\prime \prime \prime}{ }^{3} \\
& \exp \left[\mathrm{i} \boldsymbol{k}_{\perp} \boldsymbol{x}_{\perp}+\mathrm{i} \boldsymbol{k}_{\perp}^{\prime} \boldsymbol{x}_{\perp}^{\prime}+\mathrm{i} \boldsymbol{k}_{\perp}^{\prime \prime} \boldsymbol{x}_{\perp}^{\prime \prime}+\mathrm{i} \boldsymbol{k}_{\perp}^{\prime \prime \prime} \boldsymbol{x}_{\perp}^{\prime \prime \prime}\right]\left[\partial_{3}(\boldsymbol{x}) \partial_{3}\left(\boldsymbol{x}^{\prime}\right) \partial_{3}\left(\boldsymbol{x}^{\prime \prime}\right) \partial_{3}\left(\boldsymbol{x}^{\prime \prime \prime}\right)\right] \\
&\left.\exp \left[\frac{1}{2} \boldsymbol{J}^{\dagger} \mathbf{M} \boldsymbol{J}\right]\right|_{\boldsymbol{J}=0} \\
&= n_{\mathrm{e}}^{4} \int \mathrm{d} x^{3} \int \mathrm{d} x^{\prime 3} \int \mathrm{d} x^{\prime \prime 3} \int \mathrm{d} x^{\prime \prime \prime 3} \exp \left[\mathrm{i} \boldsymbol{k}_{\perp} \boldsymbol{x}_{\perp}+\mathrm{i} \boldsymbol{k}_{\perp}^{\prime} \boldsymbol{x}_{\perp}^{\prime}+\mathrm{i} \boldsymbol{k}_{\perp}^{\prime \prime} \boldsymbol{x}_{\perp}^{\prime \prime}+\mathrm{i} \boldsymbol{k}_{\perp}^{\prime \prime \prime} \boldsymbol{x}_{\perp}^{\prime \prime \prime}\right] \\
& {[\underbrace{M_{33}\left(\boldsymbol{x}^{\prime \prime \prime}-\boldsymbol{x}^{\prime \prime}\right) M_{33}\left(\boldsymbol{x}^{\prime}-\boldsymbol{x}\right)}_{\text {Part } 1}+\underbrace{M_{33}\left(\boldsymbol{x}^{\prime \prime \prime}-\boldsymbol{x}^{\prime}\right) M_{33}\left(\boldsymbol{x}^{\prime \prime}-\boldsymbol{x}\right)}_{\text {Part } 2}+\underbrace{M_{33}\left(\boldsymbol{x}^{\prime \prime \prime}-\boldsymbol{x}\right) M_{33}\left(\boldsymbol{x}^{\prime \prime}-\boldsymbol{x}^{\prime}\right)}_{\text {Part } 3}] . }
\end{aligned}
$$

Part 1 gives with $\boldsymbol{x}^{\prime \prime \prime}-\boldsymbol{x}^{\prime \prime}=\boldsymbol{r}^{\prime}$ and $\boldsymbol{x}^{\prime}-\boldsymbol{x}=\boldsymbol{r}$ :

$$
\begin{aligned}
\operatorname{Part} 1= & n_{\mathrm{e}}^{4} \int \mathrm{d} x^{3} \int \mathrm{d} x^{\prime \prime 3} \int \mathrm{d} r^{3} \int \mathrm{d} r^{\prime 3} \exp \left[\mathrm{i} \boldsymbol{x}_{\perp}^{\prime \prime}\left(\boldsymbol{k}_{\perp}^{\prime \prime}+\boldsymbol{k}_{\perp}^{\prime \prime \prime}\right)\right] \exp \left[\mathrm{i} \boldsymbol{x}_{\perp}\left(\boldsymbol{k}_{\perp}+\boldsymbol{k}_{\perp}^{\prime}\right)\right] \\
& \exp \left[\boldsymbol{i}_{\perp}^{\prime} \boldsymbol{k}_{\perp}^{\prime \prime \prime}\right] \exp \left[\mathrm{i} \boldsymbol{r}_{\perp} \boldsymbol{k}_{\perp}^{\prime}\right] M_{33}\left(\boldsymbol{r}^{\prime}\right) M_{33}(\boldsymbol{r}) \\
= & n_{\mathrm{e}}^{4}(2 \pi)^{4} \delta^{2}\left(\boldsymbol{k}_{\perp}^{\prime \prime}+\boldsymbol{k}_{\perp}^{\prime \prime \prime}\right) \delta^{2}\left(\boldsymbol{k}_{\perp}+\boldsymbol{k}_{\perp}^{\prime}\right) \int \mathrm{d} r^{3} \int \mathrm{d} r^{\prime 3} \int \frac{\mathrm{d} q^{3}}{(2 \pi)^{3}} \int \frac{\mathrm{d} q^{\prime 3}}{(2 \pi)^{3}} \\
& \exp \left[\boldsymbol{i}_{\perp}\left(\boldsymbol{k}_{\perp}^{\prime}-\boldsymbol{q}_{\perp}\right)\right] \exp \left[-\mathrm{i} r_{z} q_{z}\right] \exp \left[\mathrm{i} \boldsymbol{r}_{\perp}^{\prime}\left(\boldsymbol{k}_{\perp}^{\prime \prime \prime}-\boldsymbol{q}_{\perp}^{\prime}\right)\right] \exp \left[-\mathrm{i} r_{z}^{\prime} q_{z}^{\prime}\right] \\
& \hat{M}_{33}\left(\boldsymbol{q}^{\prime}\right) \hat{M}_{33}(\boldsymbol{q}) \\
= & n_{\mathrm{e}}^{4}(2 \pi)^{4} \delta^{2}\left(\boldsymbol{k}_{\perp}^{\prime \prime}+\boldsymbol{k}_{\perp}^{\prime \prime \prime}\right) \delta^{2}\left(\boldsymbol{k}_{\perp}+\boldsymbol{k}_{\perp}^{\prime}\right) \hat{M}_{33}\left(\boldsymbol{k}_{\perp}^{\prime \prime \prime}, 0\right) \hat{M}_{33}\left(\boldsymbol{k}_{\perp}^{\prime}, 0\right) .
\end{aligned}
$$

Parts 2 and 3 essentially provide the same if one simply adopts the definition of $\boldsymbol{r}$ and $\boldsymbol{r}^{\prime}$ as follows

Part 2: $\boldsymbol{x}^{\prime \prime \prime}-\boldsymbol{x}^{\prime}=\boldsymbol{r}^{\prime}$ and $\boldsymbol{x}^{\prime \prime}-\boldsymbol{x}=\boldsymbol{r}$,

Part 3: $\boldsymbol{x}^{\prime \prime}-\boldsymbol{x}=\boldsymbol{r}^{\prime}$ and $\boldsymbol{x}^{\prime \prime \prime}-\boldsymbol{x}=\boldsymbol{r}$.

The final result is then

$$
\begin{aligned}
\langle\phi & \left.\left(\boldsymbol{k}_{\perp}\right) \phi\left(\boldsymbol{k}_{\perp}^{\prime}\right) \phi\left(\boldsymbol{k}_{\perp}^{\prime \prime}\right) \phi\left(\boldsymbol{k}_{\perp}^{\prime \prime \prime}\right)\right\rangle_{\boldsymbol{B}}=n_{\mathrm{e}}^{4}(2 \pi)^{4}\left[\delta^{2}\left(\boldsymbol{k}_{\perp}^{\prime \prime}+\boldsymbol{k}_{\perp}^{\prime \prime \prime}\right) \delta^{2}\left(\boldsymbol{k}_{\perp}+\boldsymbol{k}_{\perp}^{\prime}\right)\right. \\
& \hat{M}_{33}\left(\boldsymbol{k}_{\perp}^{\prime \prime \prime}, 0\right) \hat{M}_{33}\left(\boldsymbol{k}_{\perp}^{\prime}, 0\right)+\delta^{2}\left(\boldsymbol{k}_{\perp}^{\prime \prime \prime}+\boldsymbol{k}_{\perp}^{\prime}\right) \delta^{2}\left(\boldsymbol{k}_{\perp}^{\prime \prime}+\boldsymbol{k}_{\perp}\right) \hat{M}_{33}\left(\boldsymbol{k}_{\perp}^{\prime \prime \prime}, 0\right) \hat{M}_{33}\left(\boldsymbol{k}_{\perp}^{\prime \prime}, 0\right) \\
& \left.+\delta^{2}\left(\boldsymbol{k}_{\perp}^{\prime \prime}+\boldsymbol{k}_{\perp}^{\prime}\right) \delta^{2}\left(\boldsymbol{k}_{\perp}^{\prime \prime \prime}+\boldsymbol{k}_{\perp}\right) \hat{M}_{33}\left(\boldsymbol{k}_{\perp}^{\prime \prime \prime}, 0\right) \hat{M}_{33}\left(\boldsymbol{k}_{\perp}^{\prime \prime}, 0\right)\right] \\
= & n_{\mathrm{e}}^{4}(2 \pi)^{4}\left[\delta^{2}\left(\boldsymbol{k}_{\perp}^{\prime \prime}+\boldsymbol{k}_{\perp}^{\prime \prime \prime}\right) \delta^{2}\left(\boldsymbol{k}_{\perp}+\boldsymbol{k}_{\perp}^{\prime}\right) \hat{M}_{\mathrm{N}}(w) \hat{M}_{\mathrm{N}}(v)+\delta^{2}\left(\boldsymbol{k}_{\perp}^{\prime \prime \prime}+\boldsymbol{k}_{\perp}^{\prime}\right) \delta^{2}\left(\boldsymbol{k}_{\perp}^{\prime \prime}+\boldsymbol{k}_{\perp}\right)\right. \\
& \left.\hat{M}_{\mathrm{N}}(w) \hat{M}_{\mathrm{N}}(u)+\delta^{2}\left(\boldsymbol{k}_{\perp}^{\prime \prime}+\boldsymbol{k}_{\perp}^{\prime}\right) \delta^{2}\left(\boldsymbol{k}_{\perp}^{\prime \prime \prime}+\boldsymbol{k}_{\perp}\right) \hat{M}_{\mathrm{N}}(w) \hat{M}_{\mathrm{N}}(u)\right] .
\end{aligned}
$$

\section{A.5. Calculation of $\left\langle I\left(\boldsymbol{k}_{\perp}\right)\right\rangle_{B}$}

$$
\begin{aligned}
\left\langle I\left(\boldsymbol{k}_{\perp}\right)\right\rangle_{\boldsymbol{B}} & =\left.\int \mathrm{d} x^{3} \exp \left[\mathrm{i} \boldsymbol{k}_{\perp} \boldsymbol{x}_{\perp}\right]\left(\partial_{1}^{2}(\boldsymbol{x})+\partial_{2}^{2}(\boldsymbol{x})\right) \exp \left[\frac{1}{2} \boldsymbol{J}^{\dagger} \mathbf{M} \boldsymbol{J}\right]\right|_{\boldsymbol{J}=0} \\
& =\int \mathrm{d} x^{3} \exp \left[\mathrm{i} \boldsymbol{k}_{\perp} \boldsymbol{x}_{\perp}\right]\left(M_{11}(\boldsymbol{x}, \boldsymbol{x})+M_{22}(\boldsymbol{x}, \boldsymbol{x})\right) \\
& =\int \mathrm{d} x^{3} \exp \left[\mathrm{i} \boldsymbol{k}_{\perp} \boldsymbol{x}_{\perp}\right]\left(M_{11}(0)+M_{22}(0)\right) \\
& =2 M_{\mathrm{N}}(0) \int \mathrm{d} x^{3} \exp \left[\mathrm{i} \boldsymbol{k}_{\perp} \boldsymbol{x}_{\perp}\right] \\
& =2(2 \pi)^{2} \delta^{2}\left(\boldsymbol{k}_{\perp}\right) L_{z} M_{\mathrm{N}}(0) \\
& =128 \pi^{4} \delta^{2}\left(\boldsymbol{k}_{\perp}\right) L_{z} \mathcal{B}\left(\frac{\beta}{2}+\frac{1}{2}, \frac{\alpha}{2}-\frac{1}{2}\right) .
\end{aligned}
$$

In the final step, $M_{\mathrm{N}}(0)$ was expressed in terms of a Beta-function $\mathcal{B}(a, b)$. This function is assigned a specific value for a given set of spectral indices $\alpha$ and $\beta$. 


$$
\begin{aligned}
\left\langle I\left(\boldsymbol{k}_{\perp}\right) I\left(\boldsymbol{k}_{\perp}^{\prime}\right)\right\rangle_{\boldsymbol{B}}=\int \mathrm{d} x^{3} \int \mathrm{d} x^{\prime 3} \exp \left[\mathrm{i}\left(\boldsymbol{k}_{\perp} \boldsymbol{x}_{\perp}+\boldsymbol{k}_{\perp}^{\prime} \boldsymbol{x}_{\perp}^{\prime}\right)\right]\left(\partial_{1}^{2}(\boldsymbol{x})+\partial_{2}^{2}(\boldsymbol{x})\right) \\
\left.\left(\partial_{1}^{2}\left(\boldsymbol{x}^{\prime}\right)+\partial_{2}^{2}\left(\boldsymbol{x}^{\prime}\right)\right) \exp \left[\frac{1}{2} \boldsymbol{J}^{\dagger} \mathbf{M} \boldsymbol{J}\right]\right|_{\boldsymbol{J}=0} \\
=\int \mathrm{d} x^{3} \int \mathrm{d} x^{\prime 3} \exp \left[i\left(\boldsymbol{k}_{\perp} \boldsymbol{x}_{\perp}+\boldsymbol{k}_{\perp}^{\prime} \boldsymbol{x}_{\perp}^{\prime}\right)\right]\left(\partial_{1}^{2}(\boldsymbol{x}) \partial_{1}^{2}\left(\boldsymbol{x}^{\prime}\right)+\partial_{1}^{2}(\boldsymbol{x}) \partial_{2}^{2}\left(\boldsymbol{x}^{\prime}\right)\right. \\
\left.\quad+\partial_{2}^{2}(\boldsymbol{x}) \partial_{1}^{2}\left(\boldsymbol{x}^{\prime}\right)+\partial_{2}^{2}(\boldsymbol{x}) \partial_{2}^{2}\left(\boldsymbol{x}^{\prime}\right)\right)\left.\exp \left[\frac{1}{2} \boldsymbol{J}^{\dagger} \mathbf{M} \boldsymbol{J}\right]\right|_{\boldsymbol{J}=0} \\
=\int \mathrm{d} x^{3} \int \mathrm{d} x^{\prime 3} \exp \left[\mathrm{i}\left(\boldsymbol{k}_{\perp} \boldsymbol{x}_{\perp}+\boldsymbol{k}_{\perp}^{\prime} \boldsymbol{x}_{\perp}^{\prime}\right)\right](\underbrace{\left(M_{11}(0)+M_{22}(0)\right)^{2}}_{\text {Part } 1}+\underbrace{2\left(M_{11}^{2}(\boldsymbol{r})+M_{22}^{2}(\boldsymbol{r})\right)+4 M_{21, \mathrm{sym}}^{2}(\boldsymbol{r})}_{\text {Part } 2}) .
\end{aligned}
$$

Part $1^{3}$ is not dependent on $\boldsymbol{r}$ and can be applied directly without difficulty:

$$
\begin{aligned}
\int \mathrm{d} x^{3} \int \mathrm{d} x^{\prime 3} \exp \left[\mathrm{i}\left(\boldsymbol{k}_{\perp} \boldsymbol{x}_{\perp}+\boldsymbol{k}_{\perp}^{\prime} \boldsymbol{x}_{\perp}^{\prime}\right)\right]\left(M_{11}(0)+M_{22}(0)\right)^{2} & =(2 \pi)^{4} \delta^{2}\left(\boldsymbol{k}_{\perp}\right) \delta^{2}\left(\boldsymbol{k}_{\perp}^{\prime}\right) L_{z} L_{z}^{\prime}\left(M_{11}(0)+M_{22}(0)\right)^{2} \\
& =(2 \pi)^{4} \delta^{2}\left(\boldsymbol{k}_{\perp}\right) \delta^{2}\left(\boldsymbol{k}_{\perp}^{\prime}\right) L_{z} L_{z}^{\prime} 4 M_{\mathrm{N}}^{2}(0)
\end{aligned}
$$

In contrast, Part 2 needs a little more work using Fourier transformations:

$$
\begin{aligned}
& \int \mathrm{d} x^{3} \int \mathrm{d} r^{3} \exp \left[\mathrm{i} \boldsymbol{x}_{\perp}\left(\boldsymbol{k}_{\perp}+\boldsymbol{k}_{\perp}^{\prime}\right)\right] \exp \left[\mathrm{i} \boldsymbol{k}_{\perp}^{\prime} \boldsymbol{r}_{\perp}\right]\left(2\left(M_{11}^{2}(\boldsymbol{r})+M_{22}^{2}(\boldsymbol{r})\right)+4 M_{21, \mathrm{sym}}^{2}(\boldsymbol{r})\right) \\
&=(2 \pi)^{2} \delta^{2}\left(\boldsymbol{k}_{\perp}+\boldsymbol{k}_{\perp}^{\prime}\right) L_{z} \int \mathrm{d} r^{3} \exp \left[\mathrm{i} \boldsymbol{k}_{\perp}^{\prime} \boldsymbol{r}_{\perp}\right]\left(2\left(M_{11}^{2}(\boldsymbol{r})+M_{22}^{2}(\boldsymbol{r})\right)+4 M_{21, \mathrm{sym}}^{2}(\boldsymbol{r})\right) \\
&=(2 \pi)^{2} \delta^{2}\left(\boldsymbol{k}_{\perp}+\boldsymbol{k}_{\perp}^{\prime}\right) L_{z} \int \mathrm{d} r^{3} \int \frac{\mathrm{d} q^{3}}{(2 \pi)^{3}} \int \frac{\mathrm{d} q^{\prime 3}}{(2 \pi)^{3}} \exp \left[\mathrm{i} \boldsymbol{k}_{\perp}^{\prime} \boldsymbol{r}_{\perp}\right] \exp \left[\mathrm{i} \boldsymbol{r}\left(\boldsymbol{q}+\boldsymbol{q}^{\prime}\right)\right] \\
&\left(2\left(\hat{M}_{11}(\boldsymbol{q}) \hat{M}_{11}\left(\boldsymbol{q}^{\prime}\right)+\hat{M}_{22}(\boldsymbol{q}) \hat{M}_{22}(\boldsymbol{q})^{\prime}\right)+4 \hat{M}_{21, \mathrm{sym}}(\boldsymbol{q}) \hat{M}_{21, \mathrm{sym}}\left(\boldsymbol{q}^{\prime}\right)\right) \\
&=(2 \pi)^{2} \delta^{2}\left(\boldsymbol{k}_{\perp}+\boldsymbol{k}_{\perp}^{\prime}\right) L_{z} \int \mathrm{d} r^{3} \int \frac{\mathrm{d} q^{3}}{(2 \pi)^{3}} \int \frac{\mathrm{d} q^{\prime 3}}{(2 \pi)^{3}} \exp \left[\mathrm{i} \boldsymbol{r}_{\perp}\left(\boldsymbol{q}_{\perp}+\boldsymbol{q}_{\perp}^{\prime}+\boldsymbol{k}_{\perp}^{\prime}\right)\right] \\
& \exp \left[\mathrm{i} r_{z}\left(q_{z}+q_{z}^{\prime}\right)\right]\left(2\left(\hat{M}_{11}(\boldsymbol{q}) \hat{M}_{11}\left(\boldsymbol{q}^{\prime}\right)+\hat{M}_{22}(\boldsymbol{q}) \hat{M}_{22}(\boldsymbol{q})^{\prime}\right)+4 \hat{M}_{21, \mathrm{sym}}(\boldsymbol{q}) \hat{M}_{21, \mathrm{sym}}\left(\boldsymbol{q}^{\prime}\right)\right) \\
&=(2 \pi)^{2} \delta^{2}\left(\boldsymbol{k}_{\perp}+\boldsymbol{k}_{\perp}^{\prime}\right) L_{z} \int \frac{\mathrm{d} q^{3}}{(2 \pi)^{3}} \int \frac{\mathrm{d} q^{\prime 3}}{(2 \pi)^{3}}(2 \pi)^{3} \delta^{2}\left(\boldsymbol{q}_{\perp}+\boldsymbol{q}_{\perp}^{\prime}+\boldsymbol{k}_{\perp}^{\prime}\right) \\
& \delta\left(q_{z}+q_{z}^{\prime}\right)\left(2\left(\hat{M}_{11}(\boldsymbol{q}) \hat{M}_{11}\left(\boldsymbol{q}^{\prime}\right)+\hat{M}_{22}(\boldsymbol{q}) \hat{M}_{22}(\boldsymbol{q})^{\prime}\right)+4 \hat{M}_{21, \mathrm{sym}}(\boldsymbol{q}) \hat{M}_{21, \mathrm{sym}}\left(\boldsymbol{q}^{\prime}\right)\right) \\
&=(2 \pi)^{2} \delta^{2}\left(\boldsymbol{k}_{\perp}+\boldsymbol{k}_{\perp}^{\prime}\right) L_{z} \int \frac{\mathrm{d} q^{3}}{(2 \pi)^{3}}\left(2\left(\hat{M}_{11}(\boldsymbol{q}) \hat{M}_{11}(\boldsymbol{a})+\hat{M}_{22}(\boldsymbol{q}) \hat{M}_{22}(\boldsymbol{a})\right)+4 \hat{M}_{21, \mathrm{sym}}(\boldsymbol{q}) \hat{M}_{21, \mathrm{sym}}(\boldsymbol{a})\right) \\
&= 8 \pi^{2} \delta^{2}\left(\boldsymbol{k}_{\perp}+\boldsymbol{k}_{\perp}^{\prime}\right) L_{z} \int \mathrm{d} q^{3} \frac{\epsilon(q) \epsilon(a)}{q^{2} a^{2}}\left[\left(1-\frac{q_{1}^{2}}{q^{2}}\right)\left(1-\frac{a_{1}^{2}}{a^{2}}\right)+\left(1-\frac{q_{2}^{2}}{q^{2}}\right)\left(1-\frac{a_{2}^{2}}{a^{2}}\right)+2\left(\frac{q_{2} q_{1}}{q^{2}}\right)\left(\frac{a_{2} a_{1}}{a^{2}}\right)\right]
\end{aligned}
$$

This integral has can be solved numerically for specific choices of the spectral indices $\alpha$ and $\beta$.

\section{A.7. Calculation of $\left\langle P(\boldsymbol{k})_{\perp}\right\rangle_{\boldsymbol{B}}$}

$$
\begin{aligned}
\left\langle P\left(\boldsymbol{k}_{\perp}\right)\right\rangle_{\boldsymbol{B}} & =\left.\int \mathrm{d} x^{3} \exp \left[\mathrm{i} \boldsymbol{k}_{\perp} \boldsymbol{x}_{\perp}\right]\left(\partial_{1}^{2}(\boldsymbol{x})-\partial_{2}^{2}(\boldsymbol{x})+2 \mathrm{i} \partial_{1}(\boldsymbol{x}) \partial_{2}(\boldsymbol{x})\right)\right|_{\boldsymbol{J}} \exp \left[\frac{1}{2} \boldsymbol{J}^{\dagger} \mathbf{M} \boldsymbol{J}\right] \\
& =\int \mathrm{d} x^{3} \exp \left[\mathrm{i} \boldsymbol{k}_{\perp} \boldsymbol{x}_{\perp}\right]\left(M_{11}^{2}(0)-M_{22}^{2}(0)+2 \mathrm{i}\left(\frac{1}{2} M_{12}(0)+\frac{1}{2} M_{21}(0)\right)\right) \\
& =0 .
\end{aligned}
$$

The last step holds because of the relations $M_{11}(0)=M_{22}(0)$ and $M_{21}(0)=M_{12}(0)=0$.

3 This is actually the same as the results obtained by computing $\left\langle I\left(\boldsymbol{k}_{\perp}\right)\right\rangle_{\boldsymbol{B}}^{2}$. 
A.8. Calculation of $\left\langle I\left(\boldsymbol{k}_{\perp}\right) P\left(\boldsymbol{k}_{\perp}^{\prime}\right)\right\rangle_{\boldsymbol{B}}$

$$
\begin{aligned}
& \left\langle I\left(\boldsymbol{k}_{\perp}\right) P\left(\boldsymbol{k}_{\perp}^{\prime}\right)\right\rangle_{\boldsymbol{B}}=\int \mathrm{d} x^{3} \int \mathrm{d} x^{\prime 3} \exp \left[\mathrm{i}\left(\boldsymbol{k}_{\perp} \boldsymbol{x}_{\perp}+\boldsymbol{k}_{\perp}^{\prime} \boldsymbol{x}_{\perp}^{\prime}\right)\right]\left(\partial_{1}^{2}(\boldsymbol{x})+\partial_{2}^{2}(\boldsymbol{x})\right) \\
& \left.\left(\partial_{1}^{2}\left(\boldsymbol{x}^{\prime}\right)-\partial_{2}^{2}\left(\boldsymbol{x}^{\prime}+2 \mathrm{i} \partial_{1}\left(\boldsymbol{x}^{\prime}\right) \partial_{2}\left(\boldsymbol{x}^{\prime}\right)\right)\right) \exp \left[\frac{1}{2} \boldsymbol{J}^{\dagger} \mathbf{M} \boldsymbol{J}\right]\right|_{\boldsymbol{J}=0} \\
& =\int \mathrm{d} x^{3} \int \mathrm{d} x^{\prime 3} \exp \left[\mathrm{i}\left(\boldsymbol{k}_{\perp} \boldsymbol{x}_{\perp}+\boldsymbol{k}_{\perp}^{\prime} \boldsymbol{x}_{\perp}^{\prime}\right)\right]\left[M_{11}^{2}(0)-M_{22}^{2}(0)+2 M_{11}^{2}(r)+2 M_{22}^{2}(r)\right. \\
& \left.+2 M_{12}^{2}(r)-2 M_{21}^{2}(r)+4 \mathrm{i} M_{21}(r)\left(M_{11}(r)+M_{22}(r)\right)\right] \\
& =(2 \pi)^{2} \delta^{2}\left(\boldsymbol{k}_{\perp}+\boldsymbol{k}_{\perp}^{\prime}\right) L_{z} \int \mathrm{d} r^{3} \int \frac{\mathrm{d} q^{3}}{(2 \pi)^{3}} \int \frac{\mathrm{d} q^{\prime 3}}{(2 \pi)^{3}} \exp \left[\mathrm{i} \boldsymbol{k}_{\perp}^{\prime} \boldsymbol{r}_{\perp}\right] \exp \left[\mathrm{i} \boldsymbol{r}\left(\boldsymbol{q}+\boldsymbol{q}^{\prime}\right)\right] \\
& {\left[+2 \hat{M}_{11}(q) \hat{M}_{11}\left(q^{\prime}\right)+2 \hat{M}_{22}(q) \hat{M}_{22}\left(q^{\prime}\right)+2 \hat{M}_{12}(q) \hat{M}_{12}\left(q^{\prime}\right)-2 \hat{M}_{21}(q) \hat{M}_{21}\left(q^{\prime}\right)\right.} \\
& \left.+4 \mathrm{i} \hat{M}_{21}\left(q^{\prime}\right)\left(\hat{M}_{11}(q)+\hat{M}_{22}(q)\right)\right] \\
& =(2 \pi)^{2} \delta^{2}\left(\boldsymbol{k}_{\perp}+\boldsymbol{k}_{\perp}^{\prime}\right) L_{z} \int \frac{\mathrm{d} q^{3}}{(2 \pi)^{3}} \int \frac{\mathrm{d} q^{\prime 3}}{(2 \pi)^{3}}(2 \pi)^{3} \delta^{2}\left(\boldsymbol{q}_{\perp}+\boldsymbol{q}_{\perp}^{\prime}+\boldsymbol{k}_{\perp}^{\prime}\right) \\
& \delta\left(q_{z}+\tilde{q}_{z}\right)\left[2 \hat{M}_{11}(q) \hat{M}_{11}\left(q^{\prime}\right)+2 \hat{M}_{22}(q) \hat{M}_{22}\left(q^{\prime}\right)+2 \hat{M}_{12}(q) \hat{M}_{12}\left(q^{\prime}\right)\right. \\
& \left.-2 \hat{M}_{21}(q) \hat{M}_{21}\left(q^{\prime}\right)+4 \mathrm{i} \hat{M}_{21}\left(q^{\prime}\right)\left(\hat{M}_{11}(q)+\hat{M}_{22}(q)\right)\right] \\
& =(2 \pi)^{2} \delta^{2}\left(\boldsymbol{k}_{\perp}+\boldsymbol{k}_{\perp}^{\prime}\right) L_{z} \int \frac{\mathrm{d} q^{3}}{(2 \pi)^{3}}\left[2 \hat{M}_{11}(q) \hat{M}_{11}(a)\right. \\
& +2 \hat{M}_{22}(q) \hat{M}_{22}(a)+2 \hat{M}_{12}(q) \hat{M}_{12}(a)-2 \hat{M}_{21}(q) \hat{M}_{21}(a) \\
& \left.+4 i \hat{M}_{21}(a)\left(\hat{M}_{11}(q)+\hat{M}_{22}(q)\right)\right] \\
& =(2 \pi)^{2} \delta^{2}\left(\boldsymbol{k}_{\perp}+\boldsymbol{k}_{\perp}^{\prime}\right) L_{z} \int \frac{\mathrm{d} q^{3}}{(2 \pi)^{3}}\left[\frac { \hat { M } _ { \mathrm { N } } ( q ) \hat { M } _ { \mathrm { N } } ( a ) } { q ^ { 2 } a ^ { 2 } } \left[2\left(q_{2}^{2}+q_{3}^{2}\right)\left(a_{2}^{2}+a_{3}^{2}\right)\right.\right. \\
& \left.+2\left(q_{1}^{2}+q_{3}^{2}\right)\left(a_{1}^{2}+a_{3}^{2}\right)-4 i\left(q_{1}^{2}+q_{2}^{2}+2 q_{3}^{2}\right) a_{2} a_{3}\right]+2 \frac{\hat{M}_{\mathrm{N}}(q) \hat{H}(a)}{q^{2} a} \\
& \left.\left[\mathrm{i} q_{1} q_{2} a_{3}-2\left(q_{1}^{2}+q_{2}^{2}+2 q_{3}^{2}\right) a_{3}\right]+\frac{\hat{M}_{\mathrm{N}}(a) \hat{H}(q)}{q^{2} a}\left[\mathrm{i} a_{1} a_{2} q_{3}\right]\right] \\
& =(2 \pi)^{2} \delta^{2}\left(\boldsymbol{k}_{\perp}+\boldsymbol{k}_{\perp}^{\prime}\right) L_{z} \int \frac{\mathrm{d} q^{3}}{(2 \pi)^{3}}\left[\frac { \hat { M } _ { \mathrm { N } } ( q ) \hat { M } _ { \mathrm { N } } ( a ) } { q ^ { 2 } a ^ { 2 } } \left[2\left(q_{2}^{2}+q_{3}^{2}\right)\left(a_{2}^{2}+a_{3}^{2}\right)\right.\right. \\
& \left.\left.+2\left(q_{1}^{2}+q_{3}^{2}\right)\left(a_{1}^{2}+a_{3}^{2}\right)\right]\right] \text {. }
\end{aligned}
$$

The last step was possible using an asymmetric property of the integrals. All terms in which $a_{3}$ occurs become zero when integrated from $-\infty$ to $\infty$. We can split up the integral into two parts covering the negative and positive regions, and it can be seen that they will cancel each other out. The remaining integral can be solved numerically for specific choices of the spectral indices $\alpha$ and $\beta$.

\section{A.9. Calculation of $\left\langle I\left(\boldsymbol{k}_{\perp}\right) \phi\left(\boldsymbol{k}_{\perp}^{\prime}\right)\right\rangle_{\boldsymbol{B}}$}

$$
\begin{aligned}
\left\langle I\left(\boldsymbol{k}_{\perp}\right) \phi\left(\boldsymbol{k}_{\perp}^{\prime}\right)\right\rangle_{\boldsymbol{B}} & =\left.n_{\mathrm{e}} a_{0} \int \mathrm{d} x^{3} \int \mathrm{d} x^{\prime 3} \exp \left[\mathrm{i} \boldsymbol{k}_{\perp} \boldsymbol{x}_{\perp}+\mathrm{i} \boldsymbol{k}_{\perp}^{\prime} \boldsymbol{x}_{\perp}^{\prime}\right]\left(\partial_{1}^{2}(\boldsymbol{x}) \partial_{3}\left(\boldsymbol{x}^{\prime}\right)+\partial_{2}^{2}(\boldsymbol{x}) \partial_{3}\left(\boldsymbol{x}^{\prime}\right)\right)\right|_{\boldsymbol{J}=0} \exp \left[\frac{1}{2} \boldsymbol{J}^{\dagger} \mathbf{M} \boldsymbol{J}\right] \\
& =0 .
\end{aligned}
$$

\section{A.10. Calculation of $\left\langle P\left(\boldsymbol{k}_{\perp}\right) \phi\left(\boldsymbol{k}_{\perp}^{\prime}\right)\right\rangle_{\boldsymbol{B}}$}

$$
\begin{aligned}
\left\langle P\left(\boldsymbol{k}_{\perp}\right) \phi\left(\boldsymbol{k}_{\perp}^{\prime}\right)\right\rangle_{\boldsymbol{B}}= & n_{\mathrm{e}} a_{0} \int \mathrm{d} x^{3} \int \mathrm{d} x^{\prime 3} \exp \left[\mathrm{i} \boldsymbol{k}_{\perp} \boldsymbol{x}_{\perp}+\mathrm{i} \boldsymbol{k}_{\perp}^{\prime} \boldsymbol{x}_{\perp}^{\prime}\right] \\
& \left.\left(\partial_{1}^{2}(\boldsymbol{x}) \partial_{3}\left(\boldsymbol{x}^{\prime}\right)-\partial_{2}^{2}(\boldsymbol{x}) \partial_{3}\left(\boldsymbol{x}^{\prime}\right)+2 \mathrm{i} \partial_{1}(\boldsymbol{x}) \partial_{2}(\boldsymbol{x}) \partial_{3}\left(\boldsymbol{x}^{\prime}\right)\right)\right|_{\boldsymbol{J}=0} \\
& \exp \left[\frac{1}{2} \boldsymbol{J}^{\dagger} \mathbf{M} \boldsymbol{J}\right] \\
= & 0 .
\end{aligned}
$$




\section{A.11. Calculation of $\left\langle I\left(\boldsymbol{k}_{\perp}\right) \phi\left(\boldsymbol{k}_{\perp}^{\prime}\right) \phi\left(\boldsymbol{k}_{\perp}^{\prime \prime}\right)\right\rangle_{\boldsymbol{B}}$}

$$
\begin{aligned}
& \left\langle I\left(\boldsymbol{k}_{\perp}\right) \phi\left(\boldsymbol{k}_{\perp}^{\prime}\right) \phi\left(\boldsymbol{k}_{\perp}^{\prime \prime}\right)\right\rangle_{\boldsymbol{B}}=\int \mathrm{d} x^{3} \int \mathrm{d} x^{\prime 3} \int \mathrm{d} x^{\prime \prime 3} \exp \left[\mathrm{i} \boldsymbol{k}_{\perp} \boldsymbol{x}_{\perp}+\mathrm{i} \boldsymbol{k}_{\perp}^{\prime} \boldsymbol{x}_{\perp}^{\prime}+\mathrm{i} \boldsymbol{k}_{\perp}^{\prime \prime} \boldsymbol{x}_{\perp}^{\prime \prime}\right] \\
& \left.\left(\partial_{1}^{2}(\boldsymbol{x}) \partial_{3}\left(\boldsymbol{x}^{\prime}\right) \partial_{3}\left(\boldsymbol{x}^{\prime \prime}\right)+\partial_{2}^{2}(\boldsymbol{x}) \partial_{3}\left(\boldsymbol{x}^{\prime}\right) \partial_{3}\left(\boldsymbol{x}^{\prime \prime}\right)\right)\right|_{\boldsymbol{J}=0} \exp \left[\frac{1}{2} \boldsymbol{J}^{\dagger} \mathbf{M} \boldsymbol{J}\right] \\
& =\int \mathrm{d} x^{3} \int \mathrm{d} x^{\prime 3} \int \mathrm{d} x^{\prime \prime 3} \exp \left[\mathrm{i} \boldsymbol{k}_{\perp} \boldsymbol{x}_{\perp}+\mathrm{i} \boldsymbol{k}_{\perp}^{\prime} \boldsymbol{x}_{\perp}^{\prime}+\mathrm{i} \boldsymbol{k}_{\perp}^{\prime \prime} \boldsymbol{x}_{\perp}^{\prime \prime}\right][\underbrace{M_{11}(0) M_{33}\left(\boldsymbol{x}^{\prime \prime}-\boldsymbol{x}^{\prime}\right)}_{\text {Part } 1} \\
& +\underbrace{2\left(M_{31}\left(\boldsymbol{x}^{\prime \prime}-\boldsymbol{x}\right) M_{31}\left(\boldsymbol{x}^{\prime}-\boldsymbol{x}\right)\right)}_{\text {Part } 2}+\underbrace{M_{22}(0) M_{33}\left(\boldsymbol{x}^{\prime \prime}-\boldsymbol{x}^{\prime}\right)}_{\text {Part } 3}+\underbrace{2\left(M_{32}\left(\boldsymbol{x}^{\prime \prime}-\boldsymbol{x}\right) M_{32}\left(\boldsymbol{x}^{\prime}-\boldsymbol{x}\right)\right)}_{\text {Part } 4}] .
\end{aligned}
$$

Parts 1 and 3 can be calculated in the same way, as they only differ in the component of $\mathbf{M}$ chosen by the derivatives $(i=1,2)$ :

$$
\begin{aligned}
& \int \mathrm{d} x^{3} \int \mathrm{d} x^{\prime 3} \int \mathrm{d} x^{\prime \prime 3} \exp \left[\mathrm{i} \boldsymbol{k}_{\perp} \boldsymbol{x}_{\perp}+\mathrm{i} \boldsymbol{k}_{\perp}^{\prime} \boldsymbol{x}_{\perp}^{\prime}+\mathrm{i} \boldsymbol{k}_{\perp}^{\prime \prime} \boldsymbol{x}_{\perp}^{\prime \prime}\right] M_{i i}(0) M_{33}\left(\boldsymbol{x}^{\prime \prime}-\boldsymbol{x}^{\prime}\right) \\
& \stackrel{\boldsymbol{x}^{\prime \prime}=\boldsymbol{x}^{\prime}+\boldsymbol{r}}{=} M_{i i}(0) \int \mathrm{d} x^{3} \int \mathrm{d} x^{\prime 3} \int \mathrm{d} r^{3} M_{33}(\boldsymbol{r}) \exp \left[\mathrm{i} \boldsymbol{x}_{\perp}^{\prime}\left(\boldsymbol{k}_{\perp}^{\prime}+\boldsymbol{k}_{\perp}^{\prime \prime}\right)\right] \exp \left[\mathrm{i} \boldsymbol{r}_{\perp} \boldsymbol{k}_{\perp}^{\prime \prime}\right] \\
& \exp \left[\mathrm{i} \boldsymbol{k}_{\perp} \boldsymbol{x}_{\perp}\right] \\
& =M_{i i}(0) L_{z}^{2}(2 \pi)^{4} \delta^{2}\left(\boldsymbol{k}_{\perp}^{\prime}+\boldsymbol{k}_{\perp}^{\prime \prime}\right) \delta^{2}\left(\boldsymbol{k}_{\perp}\right) \int \mathrm{d}^{3} r M_{33}(\boldsymbol{r}) \exp \left[\boldsymbol{i}_{\perp} \boldsymbol{k}_{\perp}^{\prime \prime}\right] \\
& =M_{i i}(0) L_{z}^{2}(2 \pi)^{4} \delta^{2}\left(\boldsymbol{k}_{\perp}^{\prime}+\boldsymbol{k}_{\perp}^{\prime \prime}\right) \delta^{2}\left(\boldsymbol{k}_{\perp}\right) \int \mathrm{d}^{3} r \int \frac{\mathrm{d} q^{3}}{(2 \pi)^{3}} \hat{M}_{33}(\boldsymbol{q}) \\
& \exp \left[\boldsymbol{i r}_{\perp}\left(\boldsymbol{k}_{\perp}^{\prime \prime}-\boldsymbol{q}_{\perp}\right)\right] \exp \left[\mathrm{i} r_{z} q_{z}\right] \\
& =M_{i i}(0) L_{z}^{2}(2 \pi)^{4} \delta^{2}\left(\boldsymbol{k}_{\perp}^{\prime}+\boldsymbol{k}_{\perp}^{\prime \prime}\right) \delta^{2}\left(\boldsymbol{k}_{\perp}\right) \int \mathrm{d} q^{3} \hat{M}_{33}(\boldsymbol{q}) \delta^{2}\left(\boldsymbol{k}_{\perp}^{\prime \prime}-\boldsymbol{q}_{\perp}\right) \delta\left(-q_{z}\right) \\
& =M_{i i}(0) L_{z}^{2}(2 \pi)^{4} \delta^{2}\left(\boldsymbol{k}_{\perp}^{\prime}+\boldsymbol{k}_{\perp}^{\prime \prime}\right) \delta^{2}\left(\boldsymbol{k}_{\perp}\right) \hat{M}_{33}\left(\boldsymbol{k}_{\perp}^{\prime \prime}, 0\right) .
\end{aligned}
$$

Equally, Parts 2 and 4 can be solved on the same basis $(i=1,2)$ :

$$
\begin{aligned}
2 & \int \mathrm{d} x^{3} \int \mathrm{d} x^{\prime 3} \int \mathrm{d} x^{\prime \prime 3} \exp \left[\mathrm{i} \boldsymbol{k}_{\perp} \boldsymbol{x}_{\perp}+\mathrm{i} \boldsymbol{k}_{\perp}^{\prime} \boldsymbol{x}_{\perp}^{\prime}+\mathrm{i} \boldsymbol{k}_{\perp}^{\prime \prime} \boldsymbol{x}_{\perp}^{\prime \prime}\right] M_{3 i}\left(\boldsymbol{x}^{\prime \prime}-\boldsymbol{x}\right) \\
& M_{3 i}\left(\boldsymbol{x}^{\prime}-\boldsymbol{x}\right) \\
= & 2 \int \mathrm{d} x^{3} \int \mathrm{d} r^{3} \int \mathrm{d} r^{\prime 3} \exp \left[\mathrm{i} \boldsymbol{x}_{\perp}\left(\boldsymbol{k}_{\perp}+\boldsymbol{k}_{\perp}^{\prime}+\boldsymbol{k}_{\perp}^{\prime \prime}\right)\right] \exp \left[\mathrm{i} \boldsymbol{r}_{\perp}^{\prime} \boldsymbol{k}_{\perp}^{\prime \prime}\right] \exp \left[\mathrm{i} \boldsymbol{r}_{\perp} \boldsymbol{k}_{\perp}^{\prime}\right] \\
& M_{3 i}\left(\boldsymbol{r}^{\prime}\right) M_{3 i}(\boldsymbol{r}) \\
= & 2 L_{z}(2 \pi)^{2} \delta^{2}\left(\boldsymbol{k}_{\perp}+\boldsymbol{k}_{\perp}^{\prime}+\boldsymbol{k}_{\perp}^{\prime \prime}\right) \int \mathrm{d} r^{3} \int \mathrm{d} r^{\prime 3} \int \frac{\mathrm{d} q^{3}}{(2 \pi)^{3}} \int \frac{\mathrm{d} q^{\prime 3}}{(2 \pi)^{3}} \exp \left[\mathrm{i} \boldsymbol{r}_{\perp}^{\prime}\left(\boldsymbol{k}_{\perp}^{\prime \prime}-\boldsymbol{q}_{\perp}^{\prime}\right)\right] \\
& \left.\exp \left[-\mathrm{i} r_{z}^{\prime} q_{z}^{\prime}\right] \exp \left[\mathrm{i} \boldsymbol{r}_{\perp}\left(\boldsymbol{k}_{\perp}^{\prime}-\boldsymbol{q}_{\perp}\right)\right] \exp \left[-\mathrm{i} r_{z} q_{z}\right] \hat{M}_{3 i}\left(\boldsymbol{q}^{\prime}\right)\right) \hat{M}_{3 i}(\boldsymbol{q}) \\
= & 2 L_{z}(2 \pi)^{2} \delta^{2}\left(\boldsymbol{k}_{\perp}+\boldsymbol{k}_{\perp}^{\prime}+\boldsymbol{k}_{\perp}^{\prime \prime}\right) \hat{M}_{3 i}\left(\boldsymbol{k}_{\perp}^{\prime \prime}, 0\right) \hat{M}_{3 i}\left(\boldsymbol{k}_{\perp}^{\prime}, 0\right) .
\end{aligned}
$$

This gives as final result:

$$
\begin{aligned}
\langle & \left.I\left(\boldsymbol{k}_{\perp}\right) \phi\left(\boldsymbol{k}_{\perp}^{\prime}\right) \phi\left(\boldsymbol{k}_{\perp}^{\prime \prime}\right)\right\rangle_{\boldsymbol{B}}=L^{2}(2 \pi)^{4} \delta^{2}\left(\boldsymbol{k}_{\perp}^{\prime}+\boldsymbol{k}_{\perp}^{\prime \prime}\right) \delta^{2}\left(\boldsymbol{k}_{\perp}\right) \hat{M}_{33}\left(\boldsymbol{k}_{\perp}^{\prime \prime}, 0\right) \\
& {\left[M_{11}(0)+M_{22}(0)\right]+2 n_{\mathrm{e}}^{2} L_{z}(2 \pi)^{2} \delta^{2}\left(\boldsymbol{k}_{\perp}+\boldsymbol{k}_{\perp}^{\prime}+\boldsymbol{k}_{\perp}^{\prime \prime}\right)\left[\hat{M}_{31}\left(\boldsymbol{k}_{\perp}^{\prime \prime}, 0\right) \hat{M}_{31}\left(\boldsymbol{k}_{\perp}^{\prime}, 0\right)\right.} \\
& \left.+\hat{M}_{32}\left(\boldsymbol{k}_{\perp}^{\prime \prime}, 0\right) \hat{M}_{32}\left(\boldsymbol{k}_{\perp}^{\prime}, 0\right)\right] \\
= & L^{2}(2 \pi)^{4} \delta^{2}\left(\boldsymbol{k}_{\perp}^{\prime}+\boldsymbol{k}_{\perp}^{\prime \prime}\right) \delta^{2}\left(\boldsymbol{k}_{\perp}\right) \hat{M}_{\mathrm{N}}(u) 2 M_{\mathrm{N}}(0) \\
& -2 n_{\mathrm{e}}^{2} L_{z}(2 \pi)^{2} \delta^{2}\left(\boldsymbol{k}_{\perp}+\boldsymbol{k}_{\perp}^{\prime}+\boldsymbol{k}_{\perp}^{\prime \prime}\right) \frac{\hat{H}(u) \hat{H}(v)}{u v}\left(u_{1} v_{1}+u_{2} v_{2}\right) .
\end{aligned}
$$

A discussion and a plot of this important result can be found in Sect. 4. 


\section{A.12. Calculation of $\left\langle P\left(\boldsymbol{k}_{\perp}\right) \phi\left(\boldsymbol{k}_{\perp}^{\prime}\right) \phi\left(\boldsymbol{k}_{\perp}^{\prime \prime}\right)\right\rangle_{\boldsymbol{B}}$}

$$
\begin{aligned}
\langle & \left.P\left(\boldsymbol{k}_{\perp}\right) \phi\left(\boldsymbol{k}_{\perp}^{\prime}\right) \phi\left(\boldsymbol{k}_{\perp}^{\prime \prime}\right)\right\rangle_{\boldsymbol{B}}=\int \mathrm{d} x^{3} \int \mathrm{d} x^{\prime 3} \int \mathrm{d} x^{\prime \prime 3} \exp \left[\mathrm{i} \boldsymbol{k}_{\perp} \boldsymbol{x}_{\perp}+\mathrm{i} \boldsymbol{k}_{\perp}^{\prime} \boldsymbol{x}_{\perp}^{\prime}+\mathrm{i} \boldsymbol{k}_{\perp}^{\prime \prime} \boldsymbol{x}_{\perp}^{\prime \prime}\right] \\
& \left.\left(\partial_{1}^{2}(\boldsymbol{x}) \partial_{3}\left(\boldsymbol{x}^{\prime}\right) \partial_{3}\left(\boldsymbol{x}^{\prime \prime}\right)-\partial_{2}^{2}(\boldsymbol{x}) \partial_{3}\left(\boldsymbol{x}^{\prime}\right) \partial_{3}\left(\boldsymbol{x}^{\prime \prime}\right)+2 \mathrm{i} \partial_{1}(\boldsymbol{x}) \partial_{2}(\boldsymbol{x}) \partial_{3}\left(\boldsymbol{x}^{\prime}\right) \partial_{3}\left(\boldsymbol{x}^{\prime \prime}\right)\right)\right|_{\boldsymbol{J}=0} \\
& \exp \left[\frac{1}{2} \boldsymbol{J}^{\dagger} \mathbf{M} \boldsymbol{J}\right] \\
= & \int \mathrm{d} x^{3} \int \mathrm{d} x^{\prime 3} \int \mathrm{d} x^{\prime \prime 3} \exp \left[\mathrm{i} \boldsymbol{k}_{\perp} \boldsymbol{x}_{\perp}+\mathrm{i} \boldsymbol{k}_{\perp}^{\prime} \boldsymbol{x}_{\perp}^{\prime}+\mathrm{i} \boldsymbol{k}_{\perp}^{\prime \prime} \boldsymbol{x}_{\perp}^{\prime \prime}\right] \\
& {\left[M_{33}\left(\boldsymbol{x}^{\prime \prime}-\boldsymbol{x}^{\prime}\right)\left(M_{11}(0)+M_{22}(0)\right)+2 M_{31}\left(\boldsymbol{x}^{\prime \prime}-\boldsymbol{x}\right) M_{31}\left(\boldsymbol{x}^{\prime}-\boldsymbol{x}\right)\right.} \\
& \left.+2 M_{32}\left(\boldsymbol{x}^{\prime \prime}-\boldsymbol{x}\right) M_{32}\left(\boldsymbol{x}^{\prime}-\boldsymbol{x}\right)+2 \mathrm{i} M_{32}\left(\boldsymbol{x}^{\prime \prime}-\boldsymbol{x}\right) M_{31}\left(\boldsymbol{x}^{\prime}-\boldsymbol{x}\right)+2 \mathrm{i} M_{32}\left(\boldsymbol{x}^{\prime}-\boldsymbol{x}\right) M_{31}\left(\boldsymbol{x}^{\prime \prime}-\boldsymbol{x}\right)\right] .
\end{aligned}
$$

This can be evaluated in exactly the same way as (A.17) because all the terms have the same basic structure. This provides:

$$
\begin{aligned}
\left\langle P\left(\boldsymbol{k}_{\perp}\right) \phi\left(\boldsymbol{k}_{\perp}^{\prime}\right) \phi\left(\boldsymbol{k}_{\perp}^{\prime \prime}\right)\right\rangle_{\boldsymbol{B}}=L^{2}(2 \pi)^{4} \delta^{2}\left(\boldsymbol{k}_{\perp}^{\prime}+\boldsymbol{k}_{\perp}^{\prime \prime}\right) \delta^{2}\left(\boldsymbol{k}_{\perp}\right) \hat{M}_{33}\left(\boldsymbol{k}_{\perp}^{\prime \prime}, 0\right)\left[M_{11}(0)+M_{22}(0)\right]+2 n_{\mathrm{e}}^{2} L_{z}(2 \pi)^{2} \delta^{2}\left(\boldsymbol{k}_{\perp}+\boldsymbol{k}_{\perp}^{\prime}+\boldsymbol{k}_{\perp}^{\prime \prime}\right) \\
{\left[\hat{M}_{31}\left(\boldsymbol{k}_{\perp}^{\prime \prime}, 0\right) \hat{M}_{31}\left(\boldsymbol{k}_{\perp}^{\prime}, 0\right)+\hat{M}_{32}\left(\boldsymbol{k}_{\perp}^{\prime \prime}, 0\right) \hat{M}_{32}\left(\boldsymbol{k}_{\perp}^{\prime}, 0\right)+i \hat{M}_{32}\left(\boldsymbol{k}_{\perp}^{\prime \prime}, 0\right) \hat{M}_{31}\left(\boldsymbol{k}_{\perp}^{\prime}, 0\right)+\mathrm{i} \hat{M}_{31}\left(\boldsymbol{k}_{\perp}^{\prime \prime}, 0\right) \hat{M}_{32}\left(\boldsymbol{k}_{\perp}^{\prime}, 0\right)\right] } \\
=+2 n_{\mathrm{e}}^{2} L_{z}(2 \pi)^{2} \delta^{2}\left(\boldsymbol{k}_{\perp}+\boldsymbol{k}_{\perp}^{\prime}+\boldsymbol{k}_{\perp}^{\prime \prime}\right) \frac{\hat{H}(u) \hat{H}(v)}{u v}\left(u_{1} v_{1}-u_{2} v_{2}+i\left(u_{1} v_{2}+u_{2} v_{1}\right)\right) .
\end{aligned}
$$

A discussion and a plot of this important result can be found in Sect. 4.

\section{Appendix B: Radio observables, synchrotron radiation, and Stokes parameters}

This section introduces the notation needed to describe radio observables. For our statistical approach, synchrotron radiation is the fundamental observed quantity on which our deduction is based. Since we are attempting to infer properties of the magnetic field statistics through statistics of the radio synchrotron observables, we need a clear and compact notation of these observables.

All accelerated charges emit electromagnetic radiation. If accelerated by a magnetic field, the radiation is called cyclotron radiation for nonrelativistic and synchrotron radiation for relativistic velocities. With regard to astrophysics the latter is far more important. This is because of the much higher power radiated by relativistic particles, since the total emitted power of an accelereated charge depends on $\gamma^{2}$, its Lorentz factor squared (Rybicki \& Lightman 1979):

$P=\frac{4}{3} \sigma_{T} c \beta^{2} \gamma^{2} \frac{B^{2}}{8 \pi}$

where $\beta=v / c$. Synchrotron radiation has a characteristic polarization, with a high percentage of linear polarization. Furthermore, the relativistic beaming effect confines the energy radiated within a cone in the direction of the moving charge. Due to these features and since a large fraction of astrophysical synchrotron emission falls into radio wavebands, it is relatively easy to detect and provides us with an excellent way to observe and study magnetic fields.

Synchrotron radiation is mainly emitted by relativistic electrons. Other charged particles like protons contribute far less to the radiated power due to their higher mass. Following Rybicki \& Lightman (1979) we assume a power-law distribution of the cosmicray electron energies with spectral index $p$

$N(\gamma) \mathrm{d} \gamma=C \gamma^{-p} \mathrm{~d} \gamma$

where $C$ is a normalization factor that determines the number density of relativistic electrons. The total power emitted per unit volume and unit frequency by these distributed electrons, assumed to have an isotropic pitch-angle distribution, is then given by the integral over $N(\gamma) \mathrm{d} \gamma$ times the single particle radiation spectrum. It can be shown that this leads to a power law in synchrotron emissivity (Rybicki \& Lightman 1979):

$j \propto \omega^{-\frac{(p-1)}{2}} B_{\perp}^{\frac{(p+1)}{2}} C$

In the end we are interested in observable quantities, namely the total and polarized intensity of the observed region. In terms of emissivity $j$, they are

total intensity

$$
\begin{aligned}
& I\left(\boldsymbol{x}_{\perp}\right)=\int \mathrm{d} z j(\boldsymbol{x}), \text { and } \\
& P\left(\boldsymbol{x}_{\perp}\right)=\int \mathrm{d} z j(\boldsymbol{x}) f(p) \exp (2 \mathrm{i} \chi(\boldsymbol{x})),
\end{aligned}
$$

where $f(p)=(p+1) /(p+7 / 3)$ is the polarization fraction, and the integrals are along the line of sight from the source to the observer. The angle $\chi$ is the polarization angle of the radiation. With $\chi$ we can introduce the effect of Faraday-rotation into our formulas. 
Faraday rotation is the rotation of the polarization plane of a linearly polarized wave in a medium with a non-scalar dielectric constant due to a magnetic field. In such an environment, the dielectric constant differs for left and right circular polarization (Rybicki \& Lightman 1979):

$\epsilon=1-\frac{\omega_{p}^{2}}{\omega\left(\omega \pm \omega_{B}\right)}$,

where $\omega$ denotes the frequency of the wave, and $\omega_{p}=e B / m c$ is the cyclotron frequency. Plus and minus signs denote the cases for right and left circular polarization, respectively. If we describe a linearly polarized wave as a superposition of a wave with right circular polarization and a wave with left circular polarization, the linear polarization plane will not remain constant.

Including Faraday rotation, the total angle $\chi$ at the location of the observer is given by

$\chi(\boldsymbol{x})=\chi_{0}(\boldsymbol{x})+\lambda^{2} \phi(\boldsymbol{x})$

Here $\chi_{0}$ denotes the polarization angle at the position of emission, whereas the Faraday depth $\phi(\boldsymbol{x})$ is defined as (Kronberg et al. 2008)

$\phi(x)=\frac{e^{3}}{2 \pi n_{\mathrm{e}}^{2} c^{4}} \int \mathrm{d} z n_{\mathrm{e}} B_{3}$.

It describes the phase angle through which the electric vector rotates by Faraday rotation. For this study, it is assumed that the electron density $n_{\mathrm{e}}$ is constant in order to simplify the calculations. Furthermore, we restrict ourselves to observations at short wavelength $\lambda$, thus ignoring the term $\exp \left(2 \mathrm{i} \lambda^{2} \phi(x)\right)$ in (B.5), which would lead to so-called intrinsic Faraday rotation. Nevertheless, $\phi$ is used as an independent radio observable, allowing us to infer information about the component of the magnetic field parallel to the line of sight. In real observations this would be possible by finding a background source to probe $\phi$ in the observed region (see Fig. 1). Sometimes we refer to the notion rotation measure (RM) instead of Faraday depth. This correctly describes the factor between the rotation angle and $\lambda^{2}$ obtained through observations. However, we use it in the context of real observations, because it is a more conventional term than Faraday depth when dealing with data.

Both $I\left(\boldsymbol{x}_{\perp}\right)$ and $P\left(\boldsymbol{x}_{\perp}\right)$ can be expressed most suitably using the Stokes parameters. After some calculations (see e.g. Rybicki \& Lightman 1979; Waelkens et al. 2009), the first three Stokes parameters $I, Q$ and $U$ can be determined for synchrotron radiation of a power-law spectrum distributed, isotropic, relativistic electron population:

$$
\begin{aligned}
& I=2 F(p) \omega^{\frac{(1-p)}{2}} \int \mathrm{d} z\left(B_{1}^{2}+B_{2}^{2}\right)^{\frac{(p-3)}{4}}\left(B_{1}^{2}+B_{2}^{2}\right) \\
& Q=2 G(p) \omega^{\frac{(1-p)}{2}} \int \mathrm{d} z\left(B_{1}^{2}+B_{2}^{2}\right)^{\frac{(p-3)}{4}}\left(B_{1}^{2}-B_{2}^{2}\right), \\
& U=2 G(p) \omega^{\frac{(1-p)}{2}} \int \mathrm{d} z\left(B_{1}^{2}+B_{2}^{2}\right)^{\frac{(p-3)}{4}} 2 B_{1} B_{2}
\end{aligned}
$$

The two functions $F(p)$ and $G(p)$ are expressed in terms of physical constants and gamma functions of the spectral index $p$ (see Waelkens et al. 2009; Enßlin \& Biermann 1998):

$$
\begin{aligned}
& F(p)=\frac{\sqrt[3]{3} e^{3}}{32 \pi^{2} m_{\mathrm{e}} c^{2}}\left(\frac{2 m_{\mathrm{e}} c}{3 e}\right)^{\frac{(1-p)}{2}} C \Gamma\left(\frac{p}{4}-\frac{1}{12}\right) \Gamma\left(\frac{p}{4}+\frac{19}{12}\right) \frac{2^{(p+1)} 2}{p+1} \\
& G(p)=\frac{\sqrt[3]{3} e^{3}}{32 \pi^{2} m_{\mathrm{e}} c^{2}}\left(\frac{2 m_{\mathrm{e}} c}{3 e}\right)^{\frac{(1-p)}{2}} C \Gamma\left(\frac{p}{4}-\frac{1}{12}\right) \Gamma\left(\frac{p}{4}+\frac{7}{12}\right) 2^{(p-3)} 2 .
\end{aligned}
$$

Throughout this study, the value $p=3$ has been adopted for the spectral index of the electron distribution. This not only simplifies calculations, it is also a reasonable choice from a physical point of view. Typical values for the spectral index of relativistic electrons in our galaxy measured directly by cosmic rays on Earth or indirectly via their synchrotron emission are around $p \approx 2.7$ (Amsler et al. 2008). Deviations from $p=3$ can be added to the results of this work later in terms of corrections.

Finally we can state $I, P$, and $\phi$ in the form to be used in this study. During our calculations, all fore factors will be suppressed for convenience, resulting in

$$
\begin{aligned}
I & =\int \mathrm{d} z\left(B_{1}^{2}+B_{2}^{2}\right), \\
P & =\int \mathrm{d} z\left(B_{1}^{2}-B_{2}^{2}+2 \mathrm{i} B_{1} B_{2}\right), \quad \text { and } \\
\phi & =\int \mathrm{d} z B_{3}
\end{aligned}
$$




\section{Appendix C: Identity of the covariance matrix with the correlation tensor for Gaussian statistics}

In Sect. 2.3 we use the fact that the covariance matrix of a Gaussian probability distribution of the magnetic field is identical to the magnetic correlation tensor. From the Gaussian probability distribution

$\mathcal{G}(B, \tilde{M})=\frac{1}{\sqrt{|2 \pi \tilde{M}|}} \exp \left[-\frac{1}{2} B^{+} \tilde{M}^{-1} B\right]$

the identity is easily to shown

$$
\begin{aligned}
M & =\left\langle B B^{\dagger}\right\rangle=\int \mathcal{D} B \mathcal{G}(B, \tilde{M}) B B^{\dagger} \\
& =\left.\left(\frac{\partial}{\partial J} \frac{\partial}{\partial J^{+}}\right)\right|_{J=0} \int \mathcal{D} B \frac{1}{\sqrt{|2 \pi \tilde{M}|}} \exp \left[-\frac{1}{2} B^{\dagger} \tilde{M}^{-1} B+J^{\dagger} B\right] \\
& =\left.\left(\frac{\partial}{\partial J} \frac{\partial}{\partial J^{\dagger}}\right)\right|_{J=0} \exp \left[\frac{1}{2} J^{\dagger} \tilde{M} J\right] \\
& =\left.\left([\tilde{M}+\tilde{M} J] \exp \left[\frac{1}{2} J^{+} \tilde{M} J\right)\right]\right|_{J=0}=\tilde{M} .
\end{aligned}
$$

In this case, we have used the generating-function technique, which is explained in Sect. 2.3.

\section{References}

Amsler, C., Doser, M., Antonelli, M., et al. 2008, Phys. Lett. B, 667, 1

Bracewell, R. N. 2000, The Fourier transform and its applications (Boston: McGraw Hill), c2000

Brandenburg, A. 2009, Plasma Physics and Controlled Fusion, 51, 124043

Brandenburg, A., \& Subramanian, K. 2005a, Phys. Rep., 417, 1

Brandenburg, A., \& Subramanian, K. 2005b, Astron. Nachr., 326, 400

Brown, J. C., Haverkorn, M., Gaensler, B. M., et al. 2007, ApJ, 663, 258

Cho, J., \& Ryu, D. 2009, ApJ, 705, L90

Eilek, J. A. 1989a, AJ, 98, 244

Eilek, J. A. 1989b, AJ, 98, 256

Enßlin, T. A. 2003, A\&A, 401, 499

Enßlin, T. A., \& Biermann, P. L. 1998, A\&A, 330, 90

Enßlin, T. A., \& Vogt, C. 2003, A\&A, 401, 835

Gabuzda, D. C. 2005, in ASP Conf. Ser., 345, ed. N. Kassim, M. Perez, W. Junor, \& P. Henning, 264

Haverkorn, M. 2007, in ASP Conf. Ser., 365, SINS - Small Ionized and Neutral Structures in the Diffuse Interstellar Medium, ed. M. Haverkorn, \& W. M. Goss, 242

Kahniashvili, T., \& Vachaspati, T. 2006, Phys. Rev. D, 73, 063507

Kronberg, P. P., Bernet, M. L., Miniati, F., et al. 2008, ApJ, 676, 70

Kuchar, P., \& Enßlin, T. A. 2011, A\&A, 529, A13

Mahmud, M., \& Gabuzda, D. C. 2008, in ASP Conf. Ser., 386, Extragalactic Jets: Theory and Observation from Radio to Gamma Ray, ed. T. A. Rector, \& D. S.

De Young, 494

Moffatt, H. K. 1978, Magnetic field generation in electrically conducting fluids

Narayan, R., \& Medvedev, M. V. 2001, ApJ, 562, L129

Oppermann, N., Junklewitz, H., Robbers, G., \& Enßlin, T. 2011, A\&A, 530, A89

Peskin, M. E., \& Schroeder, D. V. 1995, An Introduction to Quantum Field Theory, ed. M. E. Peskin, \& D. V. Schroeder (Westview Press)

Price, D. J., Bate, M. R., \& Dobbs, C. L. 2009, in Rev. Mex. Astron. Astrofis. Conf. Ser., 36, 128

Rybicki, G. B., \& Lightman, A. P. 1979, Radiative processes in astrophysics, ed. G. B. Rybicki, \& A. P. Lightman

Shukurov, A., Sokoloff, D., Subramanian, K., \& Brandenburg, A. 2006, A\&A, 448, L33

Sokoloff, D. 2007, Plasma Physics and Controlled Fusion, 49, 447

Spangler, S. R. 1982, ApJ, 261, 310

Spangler, S. R. 1983, ApJ, 271, L49

Strong, A. W., Moskalenko, I. V., \& Ptuskin, V. S. 2007, Ann. Rev. Nucl. Particle Sci., 57, 285

Subramanian, K. 2002, Bull. Astron. Soc. India, 30, 715

Taylor, A. R., Stil, J. M., \& Sunstrum, C. 2009, ApJ, 702, 1230

Vogt, C., \& Enßlin, T. A. 2005, A\&A, 434, 67

Volegova, A. A., \& Stepanov, R. A. 2010, Soviet J. Experiment. Theoret. Phys. Lett., 90, 637

Waelkens, A. H., Schekochihin, A. A., \& Enßlin, T. A. 2009, MNRAS, 398, 1970 